NBER WORKING PAPER SERIES

STORABLE VOTES AND AGENDA ORDER CONTROL. THEORY AND EXPERIMENTS

\author{
Alessandra Casella \\ Working Paper 14487 \\ http://www.nber.org/papers/w14487 \\ NATIONAL BUREAU OF ECONOMIC RESEARCH \\ 1050 Massachusetts Avenue \\ Cambridge, MA 02138 \\ November 2008
}

I thank the National Science Foundation (award \# SES-0617934) and the Guggenheim Foundation for financial support; Bogac Celen for advice and help with the experiments, Raj Advani for programming the experiments, and Shuky Ehrenberg and Uliana Popova for excellent assistance overall. Seminar audiences at the 2008 Arne Ryde Symposium on Mechanism Design, the Paris School of Economics, Columbia, Essex, and the University of Western Ontario offered valuable comments and suggestions. The views expressed herein are those of the author(s) and do not necessarily reflect the views of the National Bureau of Economic Research.

NBER working papers are circulated for discussion and comment purposes. They have not been peerreviewed or been subject to the review by the NBER Board of Directors that accompanies official NBER publications.

(C) 2008 by Alessandra Casella. All rights reserved. Short sections of text, not to exceed two paragraphs, may be quoted without explicit permission provided that full credit, including $\bigcirc$ notice, is given to the source. 
Storable Votes and Agenda Order Control. Theory and Experiments

Alessandra Casella

NBER Working Paper No. 14487

November 2008

JEL No. C9,D02,D7,D8

\begin{abstract}
$\underline{\text { ABSTRACT }}$
The paper studies a voting scheme where members of a committee voting sequentially on a known series of binary proposals are each granted a single extra bonus vote to cast as desired - a streamlined version of Storable Votes. When the order of the agenda is exogenous, a simple sufficient condition guarantees the existence of welfare gains, relative to simple majority voting. But if one of the voters controls the order of the agenda, does the scheme become less efficient? The endogeneity of the agenda gives rise to a cheap talk game, where the chair can use the order of proposals to transmit information about his priorities. The game has multiple equilibria, differing systematically in the precision of the information transmitted. The chair can indeed benefit, but the aggregate welfare effects are of ambiguous sign and very small in all parameterizations studied. The theoretical conclusions are tested through laboratory experiments. Subjects have difficulty identifying the informative strategies, and tend to cast the bonus vote on their highest intensity proposal. As a result, realized payoffs are effectively identical to what they would be if the agenda were exogenous. The bonus vote matters; the chair's control of the agenda does not.
\end{abstract}

\author{
Alessandra Casella \\ Department of Economics \\ Columbia University \\ New York, NY 10027 \\ and NBER \\ ac186@columbia.edu
}




\title{
Storable Votes and Agenda Order Control. Theory and Experiments*
}

\author{
Alessandra Casella ${ }^{\dagger}$
}

October 2008.

\begin{abstract}
The paper studies a voting scheme where members of a committee voting sequentially on a known series of binary proposals are each granted a single extra bonus vote to cast as desired - a streamlined version of Storable Votes. When the order of the agenda is exogenous, a simple sufficient condition guarantees the existence of welfare gains, relative to simple majority voting. But if one of the voters controls the order of the agenda, does the scheme become less efficient? The endogeneity of the agenda gives rise to a cheap talk game, where the chair can use the order of proposals to transmit information about his priorities. The game has multiple equilibria, differing systematically in the precision of the information transmitted. The chair can indeed benefit, but the aggregate welfare effects are of ambiguous sign and very small in all parameterizations studied. The theoretical conclusions are tested through laboratory experiments. Subjects have difficulty identifying the informative strategies, and tend to cast the bonus vote on their highest intensity proposal. As a result, realized payoffs are effectively identical to what they would be if the agenda were exogenous. The bonus vote matters; the chair's control of the agenda does not.
\end{abstract}

\section{Introduction}

Consider a group of voters faced with a series of binary decisions, each of which can either pass or fail. Decisions are taken according to the majority of votes cast, but suppose that, in addition to a regular vote for each decision, each voter is endowed with some "bonus votes" that can be spent freely over the different

*I thank the National Science Foundation (award \# SES-0617934) and the Guggenheim Foundation for financial support; Bogac Celen for advice and help with the experiments, Raj Advani for programming the experiments, and Shuky Ehrenberg and Uliana Popova for excellent assistance overall. Seminar audiences at the 2008 Arne Ryde Symposium on Mechanism Design, the Paris School of Economics, Columbia, Essex, and the University of Western Ontario offered valuable comments and suggestions.

${ }^{\dagger}$ Department of Economics, Columbia University, GREQAM, NBER and CEPR. ac186@columbia.edu 
decisions. This is the idea behind storable votes, a simple voting scheme designed to elicit and reward voters' intensity of preferences. By inducing a voter to cast more votes on decisions considered relatively more important, storable votes typically increase ex ante welfare, relative to simple majority voting.

One concern that previous work has not addressed is the potential for agenda manipulation. Because storable votes allow voters to modify their "weight" in decision-making by cumulating or reducing the number of votes they cast, control of the agenda could be particularly important. The goal of this paper is to take a first step towards the study of storable votes with endogenous agenda.

Because agenda-setting problems are famously difficult, and the storable votes game quite complex, the paper exploits two simplifications. First, the storable votes mechanism is streamlined: in addition to their regular votes, agents are endowed with a single indivisible bonus vote to be cast freely over any of the proposals. The analysis shows that when the agenda is fixed, this simplified mechanism can be sufficient to achieve welfare gains over majority voting. More precisely, the paper identifies a simple sufficient condition guaranteeing higher expected utility relative to majority voting. Welfare gains always obtain if the value of the bonus vote, relative to regular votes, is chosen correctly (in practice, is not too large), and either the number of voters is even or large enough, or the differences in intensity of preferences across proposals are important enough.

Suppose now that one of the voters assumes the role of committee chair and is granted some agenda power. The second simplification of the paper is to limit such power to the order with which decisions are brought to a vote. At the start of the game, the chair decides and announces the order of the agenda. Would the chair choose the order so as to exhaust other voters' bonus votes before presenting his own favorite proposal, and then carry it through the strength of his bonus vote, even with a narrow support and an efficiency loss? And would the efficiency loss be magnified in equilibrium, as the other committee members adjust their own strategies, possibly saving their bonus votes and failing to register their intensity of preferences? Is the efficiency comparison to simple majority voting robust to the endogenous determination of the agenda's order?

The main conclusion of the paper is that the agenda's order in this framework acquires the character of a cheap talk message: the chair is in a position to transmit information about his priorities and his planned use of the bonus vote. In line with the results of the cheap talk literature, the game has multiple equilibria that differ systematically in the precision of the information conveyed. A babbling equilibrium exists, where no information is conveyed, and the game remains identical to the exogenous agenda case. But informative equilibria also exist where, on the basis of their position in the agenda, voters are able to identify decisions over which the chair is sure not to cast his bonus vote. When only one such decision is identified, the information is very imprecise; when two are identified, more information is conveyed in equilibrium, and so on up to the fully informative equilibrium where all but one of the decisions are known not to be targets of the chair's bonus vote, and hence the one decision over which the chair casts the bonus vote is identified precisely. The ability of the chair to 
transmit information, and varying degrees of information, through the order of the agenda alone may seem a bit "too subtle" to have practical implications, but the result is quite intuitive if we interpret the cheap talk message as a norm: it is perfectly plausible to think of committees where the chair is known always to put on the table his highest priority decision first, or second, or last; or his least important decision first, or second, or last, and so on.

In equilibrium, when information is transmitted the chair effectively commits to casting his bonus vote on a subset of decisions only. The commitment is valuable because other voters refrain from competing with the chair's vote, and the chair sees his probability of being pivotal increase on the decisions he cares most about. As a result, the chair's expected utility is higher: the power to set the order of the agenda is valuable. As for the other voters, the impact of the information on their expected utility is less clear-cut: by avoiding competition with the chair, in equilibrium they face higher competition from other nonchair voters on the remaining decisions. The end result is ambiguous, as is the expected aggregate welfare effect. As for the quantitative importance of these effects, precise numerical results for all parameterizations I have studied yield very small magnitudes. Briefly then: granting control of the agenda's order to a chair need not change the game at all, if voters coordinate on the non-informative equilibrium; if the game does change, it is through the possible transmission of information, with a positive effect on the chair's expected utility, and an ambiguous effect on all other voters. Even then the best guess is that the magnitudes are very small. The welfare comparisons to simple majority voting derived under exogenous agenda carry over with little change when the chair controls the order of proposals.

But would voters be able to identify these rather subtle equilibrium strategies? And if they do not, could the chair's power in fact lead to sensible declines in efficiency? The second part of the paper reports the results of a laboratory experiment where subjects are confronted exactly with the voting game described by the model. Not too surprising, they have clear difficulties identifying the possible informative role of the agenda order, a hard task, and one made still more difficult by the multiple equilibria. Non-informative equilibrium strategies best explain the observed behaviors, and even then the strategic mistakes are higher than in the simple scenario with exogenous agenda. And yet, and this is the interesting part of the results, because subjects in great majority still cast their bonus vote on their highest intensity proposals, realized experimental payoffs track very closely the theoretical predictions of the non-informative equilibrium. In fact, because theoretical predictions are quantitatively very similar across equilibria, experimental payoffs are almost indistinguishable from equilibrium payoffs for any of the equilibria of the experimental parameterization. The theoretical analysis is confirmed: realized payoffs replicate the payoffs that would obtain if the agenda were fully exogenous. The bonus vote does matter; the chair's control of the agenda's order does not, at least in the limited form studied in this paper. Stronger conclusions will need to wait, but the paper ends with some discussion of why the insight provided by this simplest agenda game is likely to carry over to more general scenarios. 
This study is part of a larger research project studying the theoretical and experimental properties of storable votes. First proposed in Casella (2005) and studied experimentally in Casella, Gelman and Palfrey (2007) and Casella, Palfrey and Riezman (2008), storable votes resemble cumulative voting, a voting system used in US corporate boards and local jurisdictions and allowing voters to spread freely across multiple candidates a given total budget of votes. Cumulative voting is designed to increase the representation of minorities (Guinier, 1994; Issacharoff, Karlan and Pildes, 2002), and has been shown to do so effectively, theoretically (Cox, 1990), empirically (Pildes and Donoghue, 1995; Bowler, Donovan, and Brockington, 2003) and experimentally (Gerber, Morton and Rietz, 1998). It applies to single multi-candidate elections, and thus is a tool of representative democracy; storable votes instead are designed for series of binary decisions and apply to committee decision-making and direct democracy. Like cumulative voting, storable votes make it possible for a numerical minority to win occasionally, but in the case of storable votes the representation of minority interests need not be a goal in itself; it is the result of giving weight to voters' intensity of preferences, and thus it is typically efficient, in addition to being fair. From a theoretical point of view, storable votes are related to the mechanism designed by Jackson and Sonnenschein (2007), and to the almost identical voting scheme proposed independently by Hortala-Vallve (2006).

The literature on agenda games typically studies the introduction of different alternatives when a single choice must be made among several (see for example McKelvey's (1976) classic paper, Ordeshook and Palfrey (1988) for an early discussion of incomplete information, and, for more recent contributions, Dutta, Jackson and le Breton (2004) and the works discussed there). Here the problem is different because all proposals are unrelated; each of them either passes or fail, independently of the others. The possible importance of the agenda comes solely from the existence of the bonus vote. The agenda game studied in this paper recalls results and intuitions from the literature on cheap talk games, originating from Crawford and Sobel (1982). The specific model is quite different: the message comes through an action that although not costly constrains the remainder of the game; there are multiple agents, the game is sequential, and, as the analysis will show, the strategic interaction between the players depends non-trivially on the number of voters and proposals, and on the value of the bonus vote. Yet, the multiple equilibria distinguished by the coarseness of the information transmitted are clearly reminiscent of Crawford and Sobel's result. Similarly, the experiments recall experimental analyses of cheap talk games (for example, Palfrey and Rosenthal, 1991; Sopher and Zapater, 1993. See Crawford's (1998) survey and discussion) but both the design and the goals are very different. As opposed to allowing subjects to exchange messages and testing whether the messages are truthful and whether they affect the subsequent play of the game, the experiments described in this paper investigate whether subjects are aware that the order of the agenda can be used as a message, and have as goal the evaluation of the welfare properties of the voting scheme, and their robustness to strategic mistakes.

Finally, the topic invites comparison with the body of work studying infor- 
mation transmission and deliberation in committees before a decision is taken by voting (starting with Austen-Smith, 1990; see also Coughlan, 2000, AustenSmith and Feddersen, 2006, and Gerardi and Yariv, 2007). But again the focus is very different: the model of this paper is a pure private value model, where individuals differ in their preferences, as opposed to a common value model where individuals differ in their information. ${ }^{1}$ There is no deliberation, but a single agent, the chair, is allowed to send a non costly signal.

The next section describes the model; equilibrium strategies and efficiency are studied in section 3 when the agenda is exogenous, and in section 4 when the chair controls the order of the agenda; section 5 describes the experimental design and the experiment's results, and section 6 concludes. Most proofs and detailed calculations are in Appendix A; experimental instructions for one of the treatments are reproduced in Appendix B.

\section{The Model}

Consider a committee of $n$ voters, meeting to decide whether or not to implement $T$ distinct proposals, $\left\{P_{1}, . . P_{T}\right\}$, each of which can either pass or fail. The $T$ proposals constitute the known, fixed agenda of the meeting, and the committee votes over the proposals sequentially. Each member $i$ of the committee has a preference over whether any specific proposal $P_{t}$ should pass or fail and attaches some importance to having the proposal decided in his preferred direction. Both features of $i$ 's preferences - direction and intensity - are summarized by a cardinal valuation $\mathrm{v}_{i t}$. Valuations $\mathrm{v}_{i t}$ are independently and identically distributed both across proposals and across individuals according to the distribution $G(\mathrm{v})$ with support $[-1,1]$, symmetric around 0 . A negative valuation means that the voter is against the proposal; a positive valuation that he is in favor. For each proposal $P_{t}$, a committee member $i$ receives utility equal to the absolute value of his valuation $\left|\mathrm{v}_{i t}\right| \equiv v_{i t}$ if the decision goes in the preferred direction, and 0 otherwise. $^{2}$ Thus the sign of the valuation indicates the preferred outcome for the proposal, while the absolute value indicates the intensity of preferences. The utility from the entire meeting is the sum of the utilities derived from each individual proposal.

In addition to a regular vote over every proposal, each committee member is given a single indivisible bonus vote. The bonus vote is worth $B$ regular votes, where $B$ can be smaller or larger than 1 , and I will refer to $B$ as its value. When the vote over $P_{1}$ is called, each individual decides whether to vote for or against, and whether to cast 1 or $1+B$ votes. If a voter casts $1+B$ votes over $P_{1}$, he will be able to cast only his regular vote over each of the successive proposals; if instead he casts 1 vote over $P_{1}$, he retains his bonus vote for one of the successive proposals. Voting continues in this manner until the last proposal, when any

\footnotetext{
${ }^{1}$ Ordeshook and Palfrey (1988) do discuss information transmission through straw voting in a pure private value model, but, as noticed above, the focus is on a dynamic agenda game where a single choice must be made among multiple alternatives.

${ }^{2}$ Only the difference in utility between the two outcomes (passing or failing) matters.
} 
remaining bonus vote will be cast. Each proposal is decided in the direction that receives more votes; in case of a tie, a coin is flipped.

When voting over any proposal, a committee member knows his own valuations over all proposals and the set of voters still endowed with the bonus vote, a set denoted by $\Lambda_{t}$. The committee member does not know others' valuations over any of the proposals, but the independence of the valuations and the distribution $G$ from which they are drawn are common knowledge. The state of the game at $t$ is summarized by $\left(\Lambda_{t}, t\right)$.

\section{Exogenous order of proposals}

Suppose first that the proposals are voted upon in some pre-determined, publicly known order.

\subsection{Equilibrium}

The analysis focuses on symmetrical Perfect Bayesian equilibria in weakly undominated strategies. With independent valuation draws and weakly undominated strategies, all individuals vote in the direction they sincerely prefer. The only question is when to cast the bonus vote. Because the direction of the vote is always chosen sincerely, $G$ is symmetric around 0 , and valuations are independent, the decision to cast the bonus vote will depend on the valuations' intensity, not on their direction. Denote by $\boldsymbol{v}_{i}$ the vector of realized intensities for voter $i: \boldsymbol{v}_{i} \equiv\left\{v_{i 1}, \ldots, v_{i T}\right\}$, defined over support [0,1]. If we call $x_{i t}$ the number of votes cast by voter $i$ over proposal $P_{t}$, then $x_{i t}=x_{i t}\left(\boldsymbol{v}_{i}, \Lambda_{t}, G, t\right)$ where $x_{i t} \in\{1,1+B\}$ and $\sum_{t=1}^{T} x_{i t}=T+B$. At first sight, identifying equilibrium strategies may seem difficult: for a given vector $\boldsymbol{v}_{i}$ the choice to cast the bonus vote on proposal $P_{t}$ in general may depend on how many bonus votes remain available to the other voters. But when valuations are independent, the answer in fact is very simple:

Proposition 1. (1) For all $T, n, G$, and $B$ there exists an equilibrium where each voter $i$ casts his bonus vote over $P_{t}$ if and only if $v_{i t}=\max \left\{\boldsymbol{v}_{i}\right\}$. (2) This is the unique symmetrical equilibrium in weakly undominated strategies holding for all $T, n, G$, and $B$.

The proof of lemma 1 is in the Appendix, but the intuition is immediate. If everyone else follows the strategy, at any point in the game the expected number of bonus votes cast over any future proposal is identical. There is no reason to postpone or anticipate casting the bonus vote - the only consideration is the intensity of preferences. For specific values of $n, T$ and $B$ other equilibria exist where voting strategies are not responsive to intensities ${ }^{3}$; however, as the proposition states, the equilibrium identified here is the only one that exists

\footnotetext{
${ }^{3}$ For example, for $n$ odd and $B<1$ there is always an equilibrium where all voters cast their bonus vote on the same proposal $P_{t}$, for any $t \in\{1, \ldots, T\}$. The voting scheme is then identical to simple majority voting,
} 
for all parameter values. In addition, responsiveness to intensity makes the equilibrium a natural focal point.

\section{$3.2 \quad$ Efficiency}

How desirable are the welfare properties of the mechanism? In this set-up there is no reason to treat individuals asymmetrically, and a natural welfare measure is ex ante utility - expected utility before the realization of any valuation, but given $G(\mathrm{v})$ and the expectation that all voters will choose the equilibrium strategy. Call $p_{B}$ the probability of obtaining the desired outcome when casting $1+B$ votes, and $p_{1}$ the corresponding probability when casting only the regular vote, noting from Proposition 1 that, when evaluated ex ante, $p_{B t}=p_{B}$ and $p_{1 t}=p_{1}$ for all $t . E v$ is the expected value of the absolute valuation, or expected intensity, and $E v_{(k)}$ the expected $k$ th order statistic among each voter's $T$ absolute valuations (hence the $T$ th order statistics is the expected highest absolute draw). Then expected ex ante utility over the whole meeting, $E V$, is given by:

$$
E V=p_{B} E v_{(T)}+p_{1} \sum_{s=1}^{T-1} E v_{(s)}=\left(p_{B}-p_{1}\right) E v_{(T)}+T p_{1} E v
$$

To evaluate the mechanism's performance, I compare $E V$ to ex ante utility in the absence of the bonus vote, when decisions are taken by simple majority, $E W$ :

$$
E W=T p E v .
$$

The symbol $p$ indicates the probability of obtaining one's desired outcome when every voter casts a single vote:

$$
p=\sum_{z=(n+I-1) / 2}^{n+I-1}\left(\begin{array}{c}
n+I-1 \\
z
\end{array}\right)\left(\frac{1}{2}\right)^{n+I-1}
$$

where:

$$
I=\left\{\begin{array}{l}
1 \text { if } n \text { is even } \\
0 \text { if } n \text { is odd }
\end{array}\right.
$$

The Appendix shows:

Proposition 2. For all $G, T$, and $n$ there exists a range of strictly positive values for $B, \mathcal{B}(n)$, such that for all $B \in \mathcal{B}(n)$ : (i) If $n$ is even, $E V>E W$. (ii) If $n$ is odd, $E V>E W$ as long as $E v_{(T)} / E v>[T(n+1)] /[T(n-1)+2]$.

If the number of voters is even it is always possible to choose a value of $B$ that leads to a welfare improvement relative to simple majority. Not surprisingly, welfare improvements relative to simple majority are more difficult to guarantee when $n$ is odd, for the simple reason that majority voting performs much better in that case. Whether the condition in the proposition is satisfied depends on the distribution $G$, on $n$, and on $T$. Three observations are immediate. First, 
notice that $[T(n+1)] /[T(n-1)+2]$ is strictly declining in $n$ : all else equal, the larger the number of voters, the less demanding the sufficient condition, and the more likely are welfare gains. At very large $n$, there is always a value of the bonus vote that leads to welfare improvements over majority voting. ${ }^{4}$ Second, the effect of the number of proposals $T$ is less straightforward, because both $E v_{(T)} / E v$ and the ratio $[T(n+1)] /[T(n-1)+2]$ increase with $T$. Whether the condition is more easily satisfied at small or large $T$ depends on the distribution $G$. Indeed, and this is the third observation, the shape of $G$ plays a central role. Suppose for example $G(\mathrm{v})=|\mathrm{v}|^{b}=v^{b}$. Then $E v_{(T)}=T b /(T b+1)$ and $E v=b /(b+1)$. With $[T(n+1)] /[T(n-1)+2]$ strictly declining in $n$, if the condition is satisfied at $n=3$, it is satisfied at all $n$. At $n=3$ the inequality is satisfied strictly by all $b<1$, and weakly by $b=1$ (the uniform distribution). More generally, for any $T$ and $n$, the inequality is satisfied for all $b \leq(n-1) / 2$.

What is the meaning of these conditions, and why is the inequality in the proposition required? With the extra bonus vote, the probability of obtaining the desired outcome rises when the bonus vote is cast, but falls in the case of the other proposals. A welfare improvement then requires a sufficient wedge between the highest valuation and the mean valuation. The example of the power distribution makes the point clearly. The ratio $E v_{(T)} / E v$ is strictly decreasing in $b$. With $b<1$, the density function is decreasing and convex, and the probability mass is concentrated around zero. Most draws will have small values, but a draw may come from the tail of the distribution: the wedge between the expected highest intensity and mean intensity is relatively large. The higher probability of winning the vote over that one issue is valuable and overrides the lower probability of winning the remaining, relatively less important proposals. With $b>1$, on the other hand, the density function is increasing in $v$, and there is larger probability mass at higher valuations: most proposals are likely to be considered important, and the ratio $E v_{(T)} / E v$ is relatively small. Increasing one's influence on a single issue is less valuable because it comes at the cost of reduced influence over others, still likely to be considered important.

It is important to note that the critical range $\mathcal{B}(n)$ identified in the proposition depends on $n$ only: although we are assuming that $G$ is common knowledge, the mechanism does not require the planner to know the distribution of valuations. The general lesson is that, although strictly positive, $B$ should not be too large. ${ }^{5}$

Example 1. Consider the example of $G$ Uniform, and $B=1$. To evaluate more intuitively the welfare properties of storable votes and simple majority voting, express ex ante expected utility with either mechanism as share of expected first best efficiency. Expected first best efficiency $E V^{*}$ is a voter's expected utility if each proposal is decided in favor of the side with higher total valuation (the criterion that maximizes ex ante utility). Its expression, together with the ex-

\footnotetext{
${ }^{4}$ Casella and Gelman (2008) analyze in detail the scope of storable votes in large elections.

${ }^{5}$ The proof of Proposition 1 identifies thresholds for $B$ that decline monotonically with $n$. The restriction is sufficient for welfare gains, but not necessary. All numerical simulations I have run suggest that it is much stronger than needed.
} 
plicit equations for all relevant probabilities, is given in the Appendix. All three criteria, $E V, E W$, and $E V^{*}$, are scaled by a plausible lower bound: expected utility with random decision-making $E R=T E v(1 / 2)$. The normalized variables $\Omega_{B V} \equiv(E V-E R) /\left(E V^{*}-E R\right)$ and $\Omega_{N B V} \equiv(E W-E R) /\left(E V^{*}-E R\right)$ express the share of available surplus over random decision-making that the mechanism is expected to appropriate. For the representative case $T=3$ and $n \in\{2, . ., 21\}$, they are plotted in Figure 1, where the black dots correspond to storable votes, and the red dots to simple majority. In the case of simple majority, the red dots trace two distinct curves: a lower curve, corresponding to $n$ even, and a higher curve, corresponding to $n$ odd. At $n=3$, the two mechanisms are equivalent ( $n=3$ corresponds to the highest point in the figure where the black and the red dot are superimposed), but for all other $n$ storable votes outperform simple majority. The most striking aspect of the figure is the stability of the storable votes welfare measure, as opposed to the predictable sensitivity of simple majority to $n$ odd or even. As $n$ increases, the two variables stabilize to a 5 percent margin in favor of storable votes. ${ }^{6}$

\section{Endogenous order of proposals}

A frequent reaction to the storable votes mechanism is concern about its manipulability. Suppose one of the voters had the role of committee chair and set the order of the agenda. Could the chair choose the order so as to exhaust other voters' bonus votes before presenting his own favorite proposal, and then carry it through the strength of his bonus vote, even with a narrow support and an efficiency loss? And could the efficiency loss be magnified in equilibrium, as the other committee members adjust their own strategies, possibly saving their bonus votes and failing to register their intensity of preferences? This is the question studied in this section. The setting is as follows: At the start of the meeting the chair $c$ announces the order with which proposals will be brought to a vote. A voter's strategy is, for all voters, including the chair, the choice of the proposal on which to cast the bonus vote, and, for the chair alone, the order in which the votes are called.

\subsection{Equilibrium}

A first answer to the question comes immediately from the results established so far. Allowing for an endogenous order of proposals need not reduce efficiency. Call $P_{i}^{*}$ the proposal to which voter $i$ attaches highest priority: $v_{i}^{*}=\max \left\{\boldsymbol{v}_{i}\right\}$. Then:

Proposition 3. For all $T, n, G$, and $B$ there exists an equilibrium where the chair orders $P_{c}^{*}$ in any position on the agenda with equal probability, and

\footnotetext{
${ }^{6}$ The absolute improvement over randomness decreases with $n$. With $G$ symmetric, for very large $n$, random decision-making, majority voting and storable votes all approach efficiency asymptotically, although the percentage gain in favor of storable votes is maintained.
} 
every voter $i$, including the chair, casts his bonus vote over $P_{i}^{*}$. The equilibrium is identical to the exogenous agenda equilibrium of Proposition 1.

As discussed earlier, in this equilibrium the probability that a voter endowed with his bonus vote at $t$ will cast it over any of the remaining proposal is constant. From the point of view of a different committee member, then, the only criterion for casting the bonus vote is the intensity of his own preferences: there are no gains from anticipating or postponing the use of the bonus votes. The identical reasoning applies when the order of proposals is endogenous. If the proposer chooses randomly, no information about his voting strategy is conveyed; casting the bonus vote on their highest priority proposal remains a best response for all other voters, guaranteeing that a random choice is itself a best response for the proposer. The endogeneity of the proposals' order has no impact on expected outcomes.

This reasoning, however, already suggests the possibility of different equilibria where the proposer's choice of agenda order does in fact reveal information about the chair's priorities and voting strategy. Consider the following example.

Example 2. Suppose $T=2, n=3, B=1$ and $G$ Uniform. Then there exists an equilibrium where the chair $c$ orders $P_{c}^{*}=P_{1}$ and casts his bonus vote on $P_{1}$ with probability 1 ; each of the other two committee members, $j \neq c$, spends his bonus vote on $P_{1}$ if and only if $v_{1 j} \geq(3 / 2) v_{2 j}$, and thus is expected to cast the bonus vote on $P_{1}$ with probability $1 / 3$. There is also a second equilibrium, mirror-image of the first, where the chair orders $P_{c}^{*}=P_{2}$ and always casts his bonus vote on $P_{2}$; each of the other two committee members spends his bonus vote on $P_{1}$ if and only if $v_{1 j} \geq(2 / 3) v_{2 j}$, and thus is expected to cast his bonus vote on $P_{1}$ with probability $2 / 3$.

Giving control of the order of the agenda to the chair can alter the game because the agenda can be used to transmit information: the voting game becomes a cheap talk game. Through the order of the agenda, the chair can signal his priorities, and thus his voting strategy; knowing on which proposal the chair will cast his bonus vote then induces the other voters to modify their own voting strategy, supporting the chair's communication. Proposition 3 states that, as usual in cheap talk games, the uninformative "babbling" equilibrium continues to exist: there is always an equilibrium where the message is ignored and conveys no information. But the interesting question, as in example 2, is the possible existence of equilibria where information is conveyed, and their efficiency properties.

I define an equilibrium informative if, after the chair's announcement of the agenda's order, all voters identify the chair's highest priority proposal with probability higher than $1 / T$. A fully informative equilibrium is one where the chair's highest priority proposal is identified with probability 1 . We know from the literature on cheap talk games that informative equilibria exist if the interests of the player sending the message and of those receiving it are sufficiently aligned (Crawford and Sobel, 1982). In this game, establishing a general result is complicated by the fact that the commonality of interest between the chair 
and the other committee members depends on the impact of other voters' bonus votes on one's probability of being pivotal, and thus on the number of voters and proposals, and on the value of the bonus vote. It is not difficult to find examples where a fully informative equilibrium does not exist. ${ }^{7}$ But there are also important examples where a fully informative equilibrium does exist. Call $P_{c}^{*}$ the proposal to which the chair attaches highest priority: $v_{c}^{*}=\max \left\{\boldsymbol{v}_{c}\right\}$. If we restrict attention to quasi-symmetric equilibria (in weakly undominated strategies) where all voters who are not the chair play symmetric strategies, then the Appendix shows:

Proposition 4. Suppose $T=2$. (1) For all $G$ and $n$ and $B \in \mathcal{B}(n)$ there exist two payoff-equivalent fully informative equilibria such that: (i) The chair sets $P_{t}=P_{c}^{*}$ and always casts his bonus vote on $P_{t}, t \in\{1,2\}$; (ii) any other committee member $i$ casts his bonus vote on $P_{t}$ if and only if $v_{t i} \geq \alpha(n, G) v_{s i}$, $s \neq t$, where $\alpha(n, G) \geq 1$ and unique. (2) With $T=2$ and $B \in \mathcal{B}(n)$, these are the only quasi-symmetric informative equilibria holding for all $G$ and $n$.

Proposition 4 generalizes the result of Example 2 to arbitrary committee sizes and preferences distributions, as long as the value of the bonus vote falls in the set $\mathcal{B}(n)$ and there are only two proposals. The chair's relative intensity of preferences is fully transmitted by the agenda order, and the other voters' incentive to cast their bonus vote on $P_{c}^{*}$ can be reduced by the chair's commitment. ${ }^{8}$

When there are more than two proposals, an endogenous agenda allows for the existence of multiple informative equilibria that differ both in the precision of the information transmitted and in the expected payoffs, again in line with general results of cheap talk games. The chair always casts his bonus vote on his highest priority proposal; the question is which position in the agenda such proposal occupies. As in Proposition 4, there are equilibria where the proposal is known to occupy a specific position in the ordering, and thus all other committee members can predict the chair's voting strategy with probability 1 . But there are also equilibria where the chair randomizes the position of his highest intensity proposal over a subset of possible positions, and thus other voters can predict the chair's use of the bonus vote only imperfectly. We can describe these equilibria as encompassing the two cases of Propositions 3 and 4: when the subset of proposals known to include $P_{c}^{*}$ is not strict and corresponds to the full set of proposals, the equilibrium is uninformative - the babbling equilibrium of

\footnotetext{
${ }^{7} T=3, n=5, B=1 / 2$ and $G$ Uniform is one such case. Note that the definition of informative adopted here refers to revelation of the chair's underlying preferences, as opposed to predictability of the chair's strategy. It does not cover non-responsive equilibria where all voters, including the chair, cast their bonus vote on the proposal in a specific position on the agenda, regardless of intensities. As mentioned in footnote 2, such equilibria exist at these parameter values whether or not the agenda is endogenous.

${ }^{8}$ An interesting question is whether the order of proposals can be used to convey information about the chair's direction of preferences, as opposed to intensity. When preferences are independent acroos voters and proposals, revealing the direction of one's vote can be counterproductive: it induces allies to refrain from using their bonus votes on the issue, freeing their votes for future use, when their preferences may well differ from the chair's. The Appendix shows this must be the case when $n=3$.
} 
Proposition 3; when the subset corresponds to a single position, the equilibrium conveys all information - the fully informative equilibrium of Proposition 4. In all intermediate cases, when the subset is strict but larger than a single position, the equilibrium is partially informative, the precision of the information declining with the size of the subset of admissible positions. This multiplicity of equilibria differs from the "inessential" multiplicity of payoff-equivalent equilibria in Proposition 4; it is a substantive multiplicity that corresponds to different informational contents of the message ${ }^{9}$. Call $P_{i \mathcal{S}}^{*}$ the proposal to which voter $i$ attaches highest priority among some set of proposals $\mathbf{P}_{\mathcal{S}}: v_{i \mathcal{S}}^{*}=\max \left\{\boldsymbol{v}_{i \mathcal{S}}\right\}$, and $P_{i T-\mathcal{S}}^{*}$ the proposal to which voter $i$ attaches highest priority among the complement set $\mathbf{P}_{T-\mathcal{S}}$ With an eye to the parameter choices that will be made in the experiments, it is possible to show:

Proposition 5. Suppose $n \in\{3,4\}$ and $B \in \mathcal{B}(n)$. (1) For all $G$ and $T$ there exist $T$ payoff-distinct equilibria such that: (i) The chair selects his $S$-lowest intensity proposals, with $S \in\{0,1,2, . ., T-1\}$, and arranges them, in any order, in the first $S$ positions on the agenda; he orders the remaining $T-S$ proposals with equal probability in each of the last $T-S$ positions; (ii) the chair always casts his bonus vote on $P_{c}^{*}$ (and thus never casts it on the first $S$ proposals); (iii) any other committee member $j \neq c$ casts his bonus vote on $P_{j T-\mathcal{S}}^{*}$ if $v_{j T-\mathcal{S}}^{*} \geq \alpha(n, G, T, S) v_{j \mathcal{S}}^{*}$, and on $P_{j \mathcal{S}}^{*}$ otherwise. For all $G, T$, and $S, \alpha(n, G, T, S) \geq 1$ and unique. (2) These are the only payoff-distinct quasisymmetric equilibria holding for all $G, T, B \in B(n)$ and $n \in\{3,4\}$. (The proof is in the Appendix).

For future reference, note that with committees of 3 or 4 voters, $B=1$ belongs to $\mathcal{B}(n)$ : the proposition applies to environments where the bonus vote is equivalent to a regular vote. ${ }^{10}$

The proposition restates the description of the equilibria in terms of two sets of proposals, proposals in the first $S$ positions in the agenda's order, $\mathbf{P}_{\mathcal{S}}$, and proposals in the remaining $T-S$ positions, $\mathbf{P}_{T-\mathcal{S}}$. In the equilibria the proposition identifies, the chair always orders his highest intensity proposal in one of the last $T-S$ positions, choosing each of these with equal probability, and always casts his bonus vote on it. The other members of the committee best-respond to the chair's strategy by privileging proposals in the first $S$ positions as targets for their bonus vote. The larger is $S$, the smaller is the set of proposals over which the chair may cast his bonus vote, and the more informative is the equilibrium: $S=0$ corresponds to the babbling equilibrium where no information is conveyed; $S=T-1$ corresponds to the fully infor-

\footnotetext{
${ }^{9}$ See for example the discussion in Crawford (1998).

${ }^{10}$ Although not stated for $n=2$, the equilibria in the proposition hold in such a case too, with $\left.\alpha(G, T, S)\right|_{n=2}=1$ for all $G, T$, and $S$. The reason is that when $n=2$ casting the bonus vote on one's highest valuation is a dominant strategy, for any $G$ and for any $T$ and $S$ : the increase in the pivot probability associated with the use of the bonus vote is exactly identical, whether one's opponent casts the bonus vote or not. Thus whether the agenda's order is informative or not has no effect on the strategy of the voter who is not the chair. Similarly the chair can choose to transmit information through the agenda setting, or not to, with no effect on his own voting strategy. Welfare is identical to the exogenous agenda case.
} 
mative equilibrium where the chair's highest priority proposal is identified with probability 1 . The proof in the Appendix also shows that for all $T>2$, the probability with which a non-chair member of the committee casts his bonus vote on the chair's first priority proposal declines monotonically with $S$ : the more informative the equilibrium, the more successful is the chair in crowding out other voters' bonus votes. Note, as remarked by Farrell and Gibbons (1989) that the informative equilibria are substantially different from the equilibrium with exogenous agenda: through cheap talk hard information is transmitted, and strategies are supported that could not be equilibrium strategies when the order of the agenda is exogenous.

In conclusion, as in the question asked at the start of this section, it is indeed the case that setting the agenda's order gives the chair the possibility to achieve less competition with his own bonus vote. But this is an equilibrium result: the other voters are not caught unaware by the chair's strategy, nor are they tempted to counter it by hoarding votes. On the contrary, their decision to cast their bonus votes disproportionately on the other proposals is their optimal response. The question of the efficiency impact of an endogenous agenda is left open, and I turn to it now.

\subsection{Efficiency}

The existence of the non-informative equilibrium makes clear that granting control of the agenda's order to the chair need not affect efficiency in any way. But when information is transmitted, does efficiency increase, or are there other effects creating losses for the voters?

Call $E V_{i}^{S}$ the ex ante expected utility of voter $i$ in the informative equilibrium where the chair is known not to cast the bonus vote on the first $S$ proposals. With this notation, $E V_{i}^{T-1} \equiv E V_{i}^{F I}$ is $i$ 's ex ante expected utility in the fully informative equilibrium, and $E V_{i}^{0}=E V_{i}^{N I}$, $i$ 's ex ante expected utility in the non-informative equilibrium. From the perspective of the chair, the ability to reduce the competition for influence over his highest intensity proposal is indeed valuable. The Appendix shows:

Proposition 6. In all informative equilibria described in Propositions 4 and 5, $E V_{c}^{S} \geq E V_{c}^{N}$, with $S \in\{1, . ., T-1\}$.

For the chair any informative equilibrium is at least weakly superior to the non-informative equilibrium, and thus to the equilibrium with exogenous agenda. Contrary to what one might expect, however, the different informative equilibria cannot be ranked: with more precise information, the chair faces lower competition over his highest priority proposal, but more competition over proposals in the first $S$ positions of the agenda. Depending on $G, n, T$, and $S$, the gain may or may not compensate the loss, and it is not difficult to find examples where the chair's expected utility is higher in some partially informative equilibrium than in the fully informative one ${ }^{11}$.

\footnotetext{
${ }^{11}$ One such example is $n=3, B=1, G$ Uniform and $T=10$, where the chair's highest
} 
As for the other voters, the impact of the transmission of information on expected utility is predictably ambiguous. Although it allows them to reduce competition with the chair, it induces higher competition with the other committee members, all of which tend to shift their bonus votes away from the chair's highest priority proposal. How the two effects balance each other depends on the parameters of the problem. Depending on $n, T, B$ and $G$, committee members who are not chair may fare better or worse in the informative equilibria than in the non-informative equilibrium:

Examples 2 and 3. Suppose $T=2$, and $G$ Uniform. If $n=3$ and $B=1$, then $E V_{n c}^{F I}<E V_{n c}^{N}$. But if $n=5$ and $B=1 / 2$, then $E V_{n c}^{F I}>E V_{n c}^{N}$. Note that $B=1 \in \mathcal{B}(3)$ and $B=1 / 2 \in \mathcal{B}(5)$, and thus both examples are covered by Proposition $4 .{ }^{12}$

Predictably, then, the overall effect of an endogenous agenda on expected welfare is of ambiguous sign. Consider ex ante expected utility with equal probability of being chair, $E V^{S}$. Whenever the voters who are not chair benefit from the informative equilibrium, $E V^{S}>E V^{N}$; when they do not, the effect on expected welfare depends on the relative magnitude of the chair's expected gains and the other voters' expected losses. For $G$ Uniform and $B=1$, Figure 2 shows ex ante expected utility in the fully informative equilibrium, relative to expected utility with exogenous agenda and with simple majority voting, for the two cases of $n=3$ and $n=4$, as function of the number of proposals. Each plot has three series of dots: the two series of black dots plot individual expected utility for the chair (the upper one) and for a voter who is not chair (the lower one); the red dots plot expected welfare with equal probability of being chair. The upper half of the figure, Figure $2 \mathrm{a}$, reports the utility measures as share of the corresponding measures with exogenous agenda (or equivalently, in the non-informative equilibrium). Following Proposition 6 , the chair benefits in the fully informative equilibrium with both $n=3$ and $n=4$. When $n=3$, the chair's expected gain is maximal with 2 proposals and declines monotonically as the number of proposals increases; when $n=4$, the magnitude of the gain is not monotonic in $T$, reaching a maximum at 5 proposals, and declining thereafter. The expected utility of a non-chair voter mirrors imperfectly the chair's, with opposite sign: when $n=3$, the loss is maximal at $T=2$ and declines monotonically as $T$ increases; when $n=4$, the loss is maximal at $T=4$, and declines at larger $T .{ }^{13}$ Expected ex ante welfare combines the impact on the chair and on the other voters' utility and results in a small loss when $n=3$ and a small gain when $n=4$. Both individual and aggregate effects tend to disappear as the number of proposals becomes large, and the probability of competing over the same proposal declines.

expected utility corresponds to the equilibrium with $S=8$ : in choosing where to order his highest intensity proposal, the chair randomizes between the last two positions on the agenda.

${ }^{12}$ Precisely, if $n=3$ and $B=1, E V_{n c}^{F I}=0.989 E V_{n c}^{N}$; if $n=5$ and $B=1 / 2, E V_{n c}^{F I}=$ $1.0001 E V_{n c}^{N}$. Note the small quantitative effects.

${ }^{13} \mathrm{I}$ have no intuition for the non-monotonicity of expected utility gains and losses when $n=4$ - another illustration of the complicated interactions among the different parameters of the problem. 
The lower half of the figure, Figure 2b, reports the utility measures as share of the corresponding measures with simple majority voting. With $G$ Uniform and $B=1$, if $n=3$, expected utility with simple majority voting is identical to expected utility with exogenous agenda and the bonus vote, for any number of proposals, and thus the left panel of Figure $2 \mathrm{~b}$ replicates exactly the left panel of Figure 2a: in the fully informative equilibrium, the chair expects to fare better than with majority voting, the other voters expect to fare worse, and the overall effect is very slightly negative. When $n=4$, the bonus vote with exogenous agenda leads to higher expected welfare than simple majority, and the panel on the right of Figure $2 \mathrm{~b}$ shows that the welfare superiority of the bonus vote is confirmed when the agenda is endogenous and the equilibrium fully informative.

Whether positive or negative, in Figure 2 the overall effect of the endogenous agenda is small: the expected welfare change is at most of the order of two tenth of a percent, relative to welfare with exogenous agenda. This is a recurring result of all numerical examples I have studied: regardless of the distribution of intensities, of the value of the bonus vote and of the numbers of voters and proposals, I have always found the welfare impact of the informative equilibrium quantitatively small. Taken together with the existence, and empirical plausibility, of the uninformative equilibrium, the regularity suggests that the practical impact of allowing the chair to control the order of the agenda is likely to be very small, ad thus to have little effect on the welfare comparison to simple majority voting. But what happens in practice is an empirical matter, best left to experimental testing.

\section{The Experiment}

\subsection{Experimental design}

All sessions of the experiment were run at CESS, the Center for Experimental Social Science at NYU, with enrolled students recruited from the whole campus through the laboratory web site. ${ }^{14}$ No subject participated in more than one session. The main treatment variable was the proposals' order, decided either exogenously by the computer, or endogenously by one of the subjects in each group, in turn.

Subjects were matched randomly in groups of $n$ members each, where $n$ equalled 3 in some sessions and 4 in others, and were asked to vote successively over 3 proposals, named the Green, Red and Blue proposal. In addition to one regular vote for each proposal, all subjects were endowed with a single bonus vote, identical in value to the regular votes $(B=1)$. Valuations for all three proposals were drawn randomly by the computer, independently for each subject and for each proposal, and could assume any integer value between -100 and 100 (excluding 0), with equal probability. Before any decision was taken,

\footnotetext{
${ }^{14} \mathrm{~A}$ sample of the instructions from one of the sessions is given in Appendix B. The experiment used the Multistage Game software package developed jointly between the SSEL and CASSEL labs and freely available at http://research.cassel.ucla.edu/software.htm
} 
each subject was shown his or her own valuations over the three proposals. In sessions where the order of proposals was chosen exogenously, the computer selected the order randomly, independently for each group, and the monitor showed it to each group member, together with a reminder that the order had been selected by the computer. In sessions where the order was endogenous, one group member was chosen as chair and given the task of deciding the order of proposals. The order was then communicated to the other members of the chair's group, together with a reminder that it had been decided by the chair. Subjects were then asked to vote over the first proposal, and could choose whether to cast the bonus vote, or not. After the vote, the computer screen showed whether or not the proposal had passed, and the number of bonus votes still available to the other group members. Voting continued in this fashion up to the third and last proposal, where all remaining bonus votes were cast automatically. A new sequence (round) of three proposals was then repeated. At the end of each experimental session, four rounds were chosen randomly, and subjects were paid their cumulative valuation for all proposals resolved in their preferred direction in those four rounds, multiplied by a pre-determined exchange rate, in addition to a fixed show-up fee. Average earnings were about $\$ 20.00$ an hour.

The existence of multiple informative equilibria when the order of proposals is endogenous makes learning particularly important and difficult. To allow for it, groups were not rematched but remained unchanged for an entire experimental session. Because valuations were private and drawn independently, across both subjects and proposals, even with fixed group membership there was no natural scope for coordination. When the order of proposals was endogenous, the same subject acted as chair for a fixed number of rounds, specified in the instructions. Each member of a group acted as chair in turn.

The experiment was run with four different treatments: $n=3,4$ for each of two possible agenda orders, exogenous $(X)$ or endogenous $(D)$. Some of the sessions included both exogenous and endogenous order (beginning with the simpler exogenous treatment), and, as control, others did not. In sessions with both treatments, a challenge was keeping the length of the experiment reasonable, while allowing a sufficient number of rounds as chair to each group member; in sessions with exogenous order only, again the experiment had to be reasonably short to keep the subject's interest. Table 1 reports the experimental design. 
Table 1: Experimental Design

$\begin{array}{lllll}\text { Session } & \mathrm{n} & \text { order } & \text { \# Subjects } & \text { Rounds } \\ \text { s1 } & 3 & \text { X/D } & 12 & 10 / 30 \\ \text { s2 } & 3 & \text { X/D } & 15 & 10 / 30 \\ \text { s3 } & 4 & \text { X/D } & 12 & 8 / 32 \\ \text { s4 } & 4 & \text { X/D } & 16 & 5 / 20 \\ \text { s5 } & 3 & \text { X } & 12 & 20 \\ \text { s6 } & 4 & \text { X } & 12 & 12 \\ \text { s7 } & 4 & \text { X } & 12 & 12 \\ \text { s8 } & 3 & \text { D } & 9 & 18 \\ \text { s9 } & 4 & \text { D } & 12 & 20\end{array}$

Because of a programming error, in the endogenous order treatment of session s3 different members within each group were chair for a different and random number of rounds (as opposed to all being chair for 8 of the 32 rounds). The session will be singled out but I chose not to drop it because, by comparison to the other sessions, it provides insight on the influence of predictability of the chair's strategy on strategic mistakes. To verify that holding the groups fixed did not affect the results of the experiment, two of the exogenous order sessions, s6 and s7, were run with groups randomly rematching after each round of 3-proposal voting. The results from these sessions are indistinguishable from the other results.

The experiment mirrored closely the theoretical model, and thus all theoretical results apply here. With specific values for the model parameters $(n \in\{3,4\}$, $T=3, B=1, G$ Uniform), the equilibria can be characterized precisely. When the order of proposals is exogenous, there is a unique symmetrical equilibrium where every voter casts the bonus vote on his highest intensity proposal. When the order is endogenous, there are five equilibria in all: the non-informative equilibrium, replicating the exogenous order equilibrium; three payoff-equivalent full information equilibria, where the chair always orders his highest intensity proposal either first, or second, or third; and finally a single partially informative equilibrium where the chair orders his highest intensity proposal either second or third, with equal probability, and always orders first his least intensity proposal (See the discussion in the Appendix). The uniqueness of the partially informative equilibrium is particularly interesting. With three proposals, the only partially informative equilibrium corresponds to $S=1$, or the chair never casting the bonus vote on a proposal in one specific position of the agenda's order; the sequential nature of the game then ensures that the proposal on which the chair never casts the bonus vote can only be the first. ${ }^{15}$

\footnotetext{
${ }^{15}$ The intuition is not difficult to see. Suppose to the contrary that the chair were expected to order his highest intensity proposal as either $P_{1}$ or $P_{2}$, with equal probability, and thus to cast his bonus vote either on $P_{1}$ or on $P_{2}$. The best response for the other committee members is some voting strategy that treats their intensities over the first two proposals symmetrically, and compares the higher of the two to the intensity over the third proposal. Now suppose that the chair has not cast the bonus vote on $P_{1}$. Then he is certain to cast
} 
Equilibrium strategies and expected payoffs are reported in Tables $2 \mathrm{a}$ and $2 \mathrm{~b}$ for the two cases of exogenous and endogenous order respectively. As in Propositions 4 and 5 , the tables summarize the equilibrium strategy through a value $\alpha$ indicating the minimum required wedge between the intensity of the proposal receiving the bonus vote and the second highest intensity, distinguishing, where necessary, between $\alpha_{c}$, the chair's $\alpha$, and $\alpha_{n c}$, for the other voters. When the order of proposals is exogenous, $\alpha=1$ for all voters. When the order is endogenous, $\alpha_{c}=1$ always, but $\alpha_{n c}$ depends on the equilibrium. In the uninformative equilibrium, again $\alpha_{n c}=1$. In the informative equilibria, recall the distinction between $\mathbf{P}_{\mathcal{S}}$, the set of proposals known not to receive the chair's bonus vote, and the complementary set $\mathbf{P}_{\mathcal{T}-\mathcal{S}}$, and the notation $P_{i \mathcal{V}}^{*}$ indicating the proposal in some set $\mathcal{V}$ such that $v_{i \mathcal{V}}^{*}=\max \left\{\boldsymbol{v}_{i \mathcal{V}}\right\}$. Proposition 5 states that non-chair voters cast their bonus vote on $P_{j T-\mathcal{S}}^{*}$ if $v_{j T-\mathcal{S}}^{*} \geq \widetilde{\alpha}_{n c} v_{j \mathcal{S}}^{*}$, and on $P_{j \mathcal{S}}^{*}$ otherwise, for some equilibrium value $\widetilde{\alpha}_{n c}>1$. I denote such a strategy by the notation $\alpha_{n c}=\left(1, \widetilde{\alpha}_{n c}\right)$.

The expected efficiency of the voting schemes is summarized by $\Omega_{s v}\left(\Omega_{n s v}\right)$ : the expected share of available aggregate surplus appropriated with storable votes (without storable votes, i.e. with simple majority). In Table $2 \mathrm{~b}$, the different equilibria are distinguished by the superscripts. With simple majority, the endogeneity of the proposals' order is irrelevant, and efficiency is again summarized by $\Omega_{n s v}$, which is not repeated in the table. Finally, the last two columns report $E V_{c} / E V_{n c}$ (denoted in the table by $C$ ), the gain the chair is expected to reap, relative to the other members of the group, in the informative equilibria.

Table 2: Equilibrium strategies and expected payoffs.

Three proposals, $G$ Uniform, $B=1$.

Table 2a. Exogenous order of proposals.

\begin{tabular}{cccc}
$n$ & $\alpha$ & $\Omega_{s v}$ & $\Omega_{n s v}$ \\
\hline 3 & 1 & .923 & .923 \\
4 & 1 & .908 & .804
\end{tabular}

Table 2b. Endogenous order of proposals.

\begin{tabular}{cccccccccc}
$n$ & $\alpha_{c}$ & $\alpha_{n c}^{N I}$ & $\alpha_{n c}^{P I}$ & $\alpha_{n c}^{F I}$ & $\Omega_{s v}^{N I}$ & $\Omega_{s v}^{P I}$ & $\Omega_{s v}^{F I}$ & $C^{P I}$ & $C^{F I}$ \\
\hline 3 & 1 & 1 & $(1,1.17)$ & $(1,1.26)$ & .923 & .922 & .917 & 1.01 & 1.022 \\
4 & 1 & 1 & $(1,1.05)$ & $(1,1.16)$ & .908 & .9086 & .909 & 1.003 & 1.011
\end{tabular}

it on $P_{2}$. But that makes $P_{2}$ a worse target for a non-chair bonus vote than was expected ex ante: the wedge required for $v_{2 n c}$ over $v_{3 n c}$ must increase. From the perspective of the chair, the probability of a non-chair bonus vote on $P_{2}$ declines. Thus $P_{2}$ is a better target for the chair's own bonus vote than $P_{1}$; the chair cannot be indifferent and the equilibrium unravels. The Appendix proves the uniquenessof the partially informative equilibrium for the parameters used in the experimental design, but in fact the result extends to all partially informative equilibria identifed in Proposition 5. 
With this parameterization, when the order of proposals is exogenous, the bonus vote scheme is exactly as efficient as simple majority if $n=3$, and superior if $n=4$, when it is expected to appropriate a full 10 percent more of available surplus. When the order of proposals is endogenous, the uninformative equilibrium persists unchanged, while in the informative equilibria aggregate surplus as measured by $\Omega$ falls slightly when $n$ equals 3 , up to a maximum of about half a percentage point in the fully informative equilibrium, and remains effectively unchanged (more precisely, increases at most by one tenth of a percent) when $n$ equals 4 . In all informative equilibria, the change in aggregate surplus reflects a (small) improvement in expected utility for the chair, and a (small) decline for the other members of the group. Expected utility is 1 to 2 percent higher for the chair, relative to the other voters, when $n$ equals 3 , and 0.5 to 1 percent higher when $n$ equals 4 . As the number of voters increases from 3 to 4 , the deterrent effect of the chair's bonus vote declines because crowding with the other voters becomes more costly, the thresholds $\alpha_{n c}$ 's fall, and the relative advantage of the chair decreases.

\subsection{Experimental results}

The experiments have two purposes. The first is to see what strategies the subjects adopt; the second is to evaluate the efficiency of the storable votes mechanism on the basis of the realized experimental payoffs.

\subsubsection{Strategies}

The strategic behavior of the experimental subjects can be summarized briefly. Subjects have little difficulty playing the equilibrium strategy in the exogenous order treatment, but find the endogenous order treatment more challenging. They make more strategic errors, relative to both uninformative and informative equilibria, and find the latter particularly difficult. Given the multiple equilibria of the endogenous order treatment, the observation is not surprising. However, even in the presence of strategic mistakes, in all treatments they cast their bonus vote disproportionately on their highest intensity proposal.

Before describing voting choices, it is useful to discuss briefly the ordering strategies of the chairs observed in the endogenous order experiments. Figure 3 plots the fraction of all subjects who, when chair, always choose the same order of proposals, and an order that is consistent with an informative equilibrium. In figure $3 a$ the fraction is calculated over the full data set; in figure $3 \mathrm{~b}$, the results from the full data set (the foreground columns) are compared to results obtained ignoring the first two repetitions of each chair's rotation, to allow for learning (the background columns). ${ }^{16}$ The light blue columns report the fraction of chairs always casting their most intense proposal first, second or third in the agenda order. The dark blue columns report the fraction of chairs

\footnotetext{
${ }^{16}$ In Figure 3b, session s3 must be ignored when allowing for learning because of the randomness of the chair's tenure, and for consistency, it is ignored in both the foreground and background columns. Session s3 is included in figure 3a. Notice that its impact is minimal.
} 
whose strategies are always consistent with either the same fully informative ordering strategy ("max same"), or the partially informative ordering strategy ("min 1"). When chair, around 40 percent of subjects always order their highest intensity proposal in the same position (slightly less than 40 percent over the full data set, and slightly more when allowing for learning), and among them more than half order it first on the agenda. The partially informative ordering strategy, where the least intense proposal is ordered first, is less popular, followed by about 15 percent of chairs. The regularities are interesting but their interpretation is not immediate. They may reflect an understanding of the strategic role of the agenda's order, but may just as well result from a simple, plausible rule-of-thumb. More importantly, rigorously, the fully informative ordering strategy does not require that a chair always orders his highest intensity proposal in the same position over the multiple repetitions: any sequence of ordering, if understood by the other voters, is consistent with a fully informative equilibrium. ${ }^{17}$ This last observation does not apply to the partially informative equilibrium, where the equilibrium ordering is unique: any ordering where the chair's least intense proposal is not ordered first contradicts the partially informative strategy. Although of course it is also true that in all cases the chair may well alternate freely among all equilibria. The multiplicity of equilibria in the one-match, three- proposal game translates into a much larger multiplicity over the multiple matches played by each chair.

How these ambiguities are resolved matters for evaluating strategic mistakes in the other subjects' voting decisions. And because all restrictions on the set of equilibria are arbitrary, it is important to allow for a sufficient number of alternatives to build confidence in the robustness of the conclusions. I will describe the experimental data in the endogenous order treatment in terms of deviations from the following equilibria. First, the non-informative equilibrium $(N I)$ : all chairs' orderings are always interpreted as random. ${ }^{18}$ Second, the fully informative equilibrium $(F I)$ : any ordering by the chair is correctly understood as fully informative by the other voters (who therefore always know the position on the agenda of the chair's most intense proposal). Third, an intermediate, more realistic scenario: a chair' ordering is interpreted as random unless the chair always casts his or her most intense proposal in the same position, in which case it is understood as fully informative $(F I / N I)$. Finally, a fourth scenario allowing for partially informative strategies: a chair' ordering is interpreted as random unless the chair always casts his or her least intense proposal first, in which case the ordering is understood as partially informative $(P I / N I)$. The equilibria are identified by the ordering strategy of the chair, and given such a strategy, the other subjects' equilibrium behavior is pinned down uniquely. Notice that no ordering decision by the chair can ever be interpreted as a strategic mistake. ${ }^{19}$

\footnotetext{
${ }^{17}$ Most naturally, some repeated pattern: for example, the chair casts the most intense proposal first, then second, then third, and then again first, etc. But in fact, as stated in the text, any sequence of ordering is consistent with a fully informative equilibrium.

${ }^{18}$ If the chair's ordering is interpreted as random, any actual ordering by the chair is a best response.

${ }^{19}$ In contrast, the repetition at all matches of the partially informative equilibrium specifies
} 
I classify as an "error" each round of play (each three-proposal game) where a subject does not cast the bonus vote on the proposal identified by his equilibrium strategy. Figure 4a reports the aggregate error rate - the total number of errors divided by the total number of rounds played by all subjects - for each experimental session, on the horizontal axis, and the experimental treatment and equilibrium on the vertical axis. The lowest series of points, with vertical coordinates indicated by $X$, corresponds to the exogenous order treatment; the remaining series corresponds to the different equilibria of the endogenous order treatment just discussed, starting with the non-informative equilibrium $(D-N I)$, up to the top-most series, the fully informative equilibrium $(D-F I)$. Yellow symbols refer to groups of 4 voters, black symbols to groups of 3 voters and the single red symbol to the 4 -voter session s3, where chairs alternated randomly; squares refer to the two $D$-only treatments.

With predictable variation across sessions, Figure 4a shows that error rates tend to be lowest in the exogenous order treatment. In the endogenous order treatment they are lowest when calculated relative to the non-informative equilibrium, and highest when calculated relative to fully informative equilibrium strategies. All but one $D-F I$ error rates and half of all $D-N I$ error rates are higher than all $X$ error rates. Errors relative to $P I / N I$ and $F I / N I$ equilibria are intermediate, reflecting the high frequency of chairs classified as playing noninformative ordering strategies. The size of the group appears not to matter in the exogenous treatment: there is no systematic difference between $n=3$ and $n=4$ sessions; whether the conclusion holds in the endogenous treatment too is less clear. Session s3 is not an outlier, casting additional doubts on the importance of the chair's ordering strategy in influencing voting choices. Predictably, errors are particularly high in the $D$-only sessions, indicated by the squares.

This last observation suggests that it may be important to check for learning effects. If, plausibly, learning is harder in the endogenous order treatment, the disparity in error rates between the two treatments could disappear when allowing for learning. Figure $4 \mathrm{~b}$ reports error rates calculated over three different subsamples of the data, to allow for three different types of learning. The highest hurdle of the endogenous order equilibria is predicting each chair's ordering strategy, and the first way to allow for learning is to ignore the first two matches of each chair's tenure (and, for consistency, the first two matches of the exogenous order treatment). The results from these data are plotted in the left-most diagram in Figure 4b, with label "Learning 1". ${ }^{20}$ Alternatively, we can think of learning simply as adapting to the experimental setting and the computer program, as is standard in most experiments, and we can control for it by ignoring

the chair's ordering strategy too, and because such ordering is in fact not observed frequently in the data - about 45percent of the times, averaging over all chairs and sessions - the overall number of strategic deviations, including both ordering and voting choices, is much higher. Thus, because the requirements are more stringent, the partially informative equilibrium, played over all repetitions, is a worse description of the data than any of the alternatives described above, and I ignore it below in the discussion of observed strategies.

${ }^{20}$ In this subsample, the data from session $\mathrm{s} 4$ are dropped, because dropping the first two matches of each chair's tenure is meaningless when subjects do not play the role of chair in fixed rotations. 
the first five rounds of each experimental session. The resulting error rates are plotted in the central diagram of Figure 4b, with label "Learning 2". But ignoring the first rounds of each session affects the endogenous order treatment only in the $D$-only sessions. Ignoring the first five rounds of each treatment, as opposed to each experimental session, is a better alternative and addresses the difficulty of learning equilibrium strategies (the third diagram in figure $4 \mathrm{~b}$, with label "Learning 3"). Although allowing for the first type of learning improves the relative performance of the $F I / N I$ strategies, in all three diagrams error rates continue to be lowest in the exogenous order treatment; within the endogenous order treatment they continue to be lowest when calculated relative to the non-informative equilibrium, and highest relative to the fully informative equilibrium. The conclusions are unchanged, and I ignore learning in what follows, reporting results for the full data set. Because the results from session s3 are not systematically different from the other results they will not appear with a different symbols in the rest of the figures.

Figure 4 presents aggregate error rates, but a natural question is how much heterogeneity in individual behavior is hidden by the aggregation. Figure 5 reports histograms of individual error rates, by treatment and equilibrium. Each column represents the fraction of all subjects in the given treatment whose individual error rate, in percentage terms, falls in the corresponding bin. In both treatments, there is a fair amount of heterogeneity in behavior, but the real outliers are few, and the differences in aggregate error rates are a fair mirror of individual behavior. In the exogenous order treatment, 77 percent of all subjects have error rates below 10 percent; when the order is endogenous; that fraction falls to 68 percent if we consider uninformative equilibrium strategies, and 45 percent if we consider fully informative strategies. At the other extreme, 5 percent of all subjects have error rates above 30 percent when the order is exogenous, but 12 to 13 percent do so when the order is endogenous, with little variation across the different reference equilibria. ${ }^{21}$ Notice that although strategic mistakes increase, even in the endogenous order treatment almost 70 percent of subjects cast their bonus vote on their highest priority proposal more than 90 percent of the times. It is this continued sensitivity of storable votes to subjects' intensity of preferences that explains the results of the following section.

\subsubsection{Payoffs.}

The relative difficulty of the endogenous order treatment for the experimental subjects is not surprising: the multiple equilibria, and the subtleties of the informative strategies are sufficient causes for confusion. The real interest of the experiments is in asking how efficient the voting mechanism can be in the presence of these strategic errors. In particular, are concerns about the chair's ability to manipulate the voting mechanism against the common good justified

\footnotetext{
${ }^{21}$ Because individual deviations from equilibrium strategies are reported as error rates, as opposed to absolute numbers, they tend to be higher when the relevant number of rounds is lower. Thus the measure penalizes the exogenous order treatment.
} 
when voters deviate from equilibrium strategies? How good a predictor of actual outcomes can theory be then? In past experiments with exogenous agenda and multiple bonus votes, subjects typically made strategic mistakes, and yet succeeded in appropriating experimental payoffs that were remarkably close to the theoretical predictions (Casella, Gelman and Palfrey, 2006; Casella, Palfrey and Riezman, 2008). A similar conclusion holds in the present set of experiments.

Figure 6 plots, for both treatments, realized aggregate experimental payoffs in each session and compares them to the corresponding payoffs had the subjects played the equilibrium strategies (figure 6a), or in the absence of the bonus vote (figure $6 \mathrm{~b}$ ). Payoffs are expressed as normalized share of available surplus, $\Omega$, and all payoffs, whether with equilibrium strategies, with simple majority, or maximizing the ex post aggregate surplus are calculated on the basis of the realized experimental draws. Different colors are used for $n=3$ (black) and $n=4$ (yellow) experiments, and the 45 degree lines make the figure easy to read.

The panels of figure 6a report experimental payoffs on the vertical axis versus equilibrium payoffs, on the horizontal axis. The upper left panel shows results for the exogenous agenda treatment. The wider dispersion of the yellow dots, both along and around the 45 degree line, reflects the much lower number of $n=4$ matches, and thus the higher variability of average intensities and aggregate payoffs. The experimental results match the theory very closely: as predicted, the share of surplus appropriated by the subjects tends to be higher for groups of 3 voters than for groups of 4 , and in both cases the distance from the 45 degree line is small, indicating that the experimental results deviate little from the payoffs that subjects would have obtained had they all played equilibrium strategies.

The remaining panels of figure $6 \mathrm{a}$ report the results from the endogenous agenda treatment. The question is how good a predictor theory is, and thus it is natural to compare aggregate experimental payoffs, or equivalently mean payoffs, to the three payoff-distinct theoretical equilibria (NI,PI and FI), without concerns for the changing identity of the chairs, the repetitions of the game, and the strategic difficulties experienced by the subjects. ${ }^{22}$ In the endogenous order treatment, the number of matches with 3 or 4-member groups is comparable, and there is no difference in the dispersion of payoffs for the two types of experiments. Again, the experimental outcomes replicate the equilibrium results and lie either on or very close to the 45 degree line. Surprisingly, this is true when evaluated both relative to the uninformative and to the informative equilibria, including the partially informative equilibrium, regardless of the larger number of strategic mistakes. ${ }^{23}$

Figure 6b compares experimental payoffs, on the vertical axis, to what they would have been with simple majority voting, given the experimental draws.

\footnotetext{
${ }^{22}$ In any case, as the figures imply, plots comparing aggregate payoffs to $P I / N I$ and $F I / N I$ equilibrium payoffs are effectively identical.

${ }^{23}$ In the plot comparing experimental payoffs to the informative equilibrium payoffs, the dots indicating sessions $s 3$ and $s 4$ are superimposed and appear as one (the higher of the two yellow $n=4$ dots).
} 
The first panel, on the left, reports the results for the exogenous order treatment: as predicted, in 3-member groups the experimental payoffs are effectively identical to what they would have been without the bonus vote; they are substantially higher in 4-member groups, again the dispersion reflecting the lower number of $n=4$ experimental matches. The predictive power of the theory is confirmed for the endogenous order sessions: compared to majority voting, aggregate payoffs with storable votes are very similar, possibly just slightly inferior, for 3-member groups, and clearly superior for 4-member groups. From a practical perspective, the comparison of the experimental results to majority voting is the important result of the paper. It shows both that storable votes do indeed result in different outcomes: the bonus vote does matter, and that, in the experiments at least, the endogeneity of the agenda order does not compromise storable votes' welfare properties.

The larger number of strategic errors in the endogenous order treatment, particularly but not only relative to informative equilibria, is not mirrored by a noticeable decline in realized payoffs. The reason is that although subjects fail to grasp the subtleties of equilibrium strategies, for the most part they continue to cast the bonus vote on high intensity proposals. Thus the bonus vote continues to give weight to the voters' intensity of preferences; it is this aspect of the voting strategies that goes to the core of the voting mechanism and is mostly reflected in realized payoffs. As mentioned earlier, the robustness of the efficiency results is a recurring feature of storable votes experiments.

Finally, did the chair succeeds in appropriating a larger share of the surplus, relative to the other subjects? The theory predicts this would hold in the informative equilibria only, but even then the quantitative effect should be small. By isolating the returns to the chair, the sample size is reduced to one third (for $n=3$ sessions) or one fourth $(n=4)$ of the original size, and the sampling variability trumps the differences in strategies. More insight can be gained by aggregating the data over all sessions where groups have equal size, and reducing the noise. In the $n=4$ experiments, relative to other committee members, chairs did better than they would have done in any equilibrium, whether informative or uninformative, and better than with simple majority: the strategic errors favored the chairs. The gain however was always small - averaging over all subjects, relative to a non-chair voter, a chair increased his payoff by between 0.4 and 0.6 percent in all cases. In the $n=3$ experiments, relative to nonchairs, the chairs did better than they would have done in the non-informative equilibrium, but worse than in the informative equilibria and worse than with simple majority. Here the potentially larger gain available to the chair in the informative equilibrium is compromised by the strategic errors. But again the quantitative effects are always very small, always smaller than 1 percent. In the experiments, any distributional effect is minor, if it exists at all. 


\section{Conclusions}

Storable votes are designed to elicit and reward each voter's relative intensity of preferences, and thus increase the efficiency of decision-making and the representation of the minority. One important concern however, is whether the possibility to shift votes across decision makes storable votes particularly sensitive to agenda manipulation. This paper studies a scenario where the set of decisions to be voted upon is fixed, but the chair controls the order with which they are called to a vote. The game is then transformed into a cheap talk game, where the chair can use the order of the agenda to transmit information about his priorities. In line with the results of the cheap talk literature, several equilibria are possible, distinguished by the precision with which the information is conveyed. When information is transmitted, it discourages the other voters from competing with the chair, and increases the chair's expected payoff. The effect on the other voters' utility and aggregate welfare is ambiguous, and in all parameterizations studied quantitatively very small.

In laboratory experiments, subjects have clear difficulties in identifying the informative equilibria: while the individual chairs' ordering strategy is reasonably consistent, the informational content of the chair's strategy is not recognized by the other group members. Nevertheless, because bonus votes are disproportionately cast on hi intensity proposals, the experimental payoffs are remarkably close to the theoretical predictions, a robustness result already observed in past storable votes experiments with exogenous agendas. In this paper, the theoretical predictions are that the chair's control of the order of the agenda should have a negligible effect on the welfare properties of storable votes. And this is indeed the final conclusion of the experiments: the bonus vote does matter; the chair's control of the agenda does not.

It is true, however, that the agenda power granted to the chair is minimal. Even restricting the chair to control the order of the proposals, suppose the order was announced sequentially, after observing how many votes have been cast, as opposed to being chosen once and for all at the beginning of the game. Would results change? Some equilibria of the game studied in this paper remain equilibria here: the babbling equilibrium, where no information is conveyed, the fully informative equilibrium where the chair always calls his highest intensity proposal first, and the partially informative equilibrium where the chair never calls his highest intensity proposal first. In general the other equilibria break down because the uncertainty over the agenda's order prevents non-chair voters from comparing their intensities over decisions over which the chair's bonus vote is expected, or not expected; other equilibria may well appear. But the game remains essentially a game of information transmission, an interesting but, in this setting, very subtle role for agenda control. At least in the equilibria that carry over from this paper, the differences in expected outcomes with respect to an exogenous agenda remain minor.

How about the more interesting case where the chair controls not only the order but also the content of the agenda? A plausible model would describe voters facing a set of possible decisions, only a subset of which would be voted 
upon, with the chair choosing sequentially the next decision to be brought to the table. I leave this problem for future work, but some preliminary thoughts suggest that the insights derived from this paper may carry over. The difficult problem facing voters who are not chair is the uncertainty over which decisions will be presented in the future. But this uncertainty is present even when the sequential agenda is exogenous - it is a different problem from protecting oneself from possible manipulations by the chair. Where agenda power matters is once again in granting the chair the possibility to transmit information about his priorities.

\section{References}

1. Austen-Smith, David, 1990, "Information Transmission in Debate", American Journal of Political Science, 34, 124-152.

2. Austen-Smith, David, and Timothy Feddersen, 2006, "Deliberation, Preference Uncertainty and Voting Rules," American Political Science Review,

3. Bowler, Shaun, Todd Donovan, and David Brockington, 2003. Electoral Reform and Minority Representation: Local Experiments with Alternative Elections. Columbus: Ohio State University Press.

4. Casella, Alessandra, 2005. "Storable Votes." Games and Economic Behavior 51:391-419.

5. Casella, Alessandra and Andrew Gelman, 2008, "A Simple Scheme to Improve the Efficiency of Referenda", Journal of Public Economics, 92:22402261.

6. Casella, Alessandra, Andrew Gelman and Thomas R. Palfrey, 2006. "An Experimental Study of Storable Votes", Games and Economic Behavior $57: 123-154$.

7. Casella, Alessandra, Thomas R. Palfrey, and Raimond Riezman, 2008. "Minorities and Storable Votes", Quarterly Journal of Political Science, $3: 165-200$.

8. Coughlan, Peter J., 2000, "In Defense of Unanimous Jury Verdicts: Mistrials, Communication, and Strategic Voting", American Political Science Review, 94:375-393.

9. Cox, Gary. 1990, "Centripetal and Centrifugal Incentives in Electoral Systems", American Journal of Political Science. 34:903-935.

10. Crawford, Vincent and Joel Sobel, 1982, "Strategic Information Transmission", Econometrica, 50:1431-1452.

11. Crawford, Vincent, 1998, "A Survey of Experiments on Communication via Cheap Talk", Journal of Economic Theory, 78:286-298. 
12. Dutta, Bhaskar, Matthew Jackson, and Michel le Breton, 2004, "Equilibrium Agenda Formation", Social Choice and Welfare, 23:21-57.

13. Farrell, Joseph, 1995, "Talk is Cheap", American Economic Review, 85:186190.

14. Farrell, Joseph and Robert Gibbons, 1989, "Cheap Talk Can Matter in Bargaining", Journal of Economic Theory, 48:221-237.

15. Gerardi, Dino and Leeat Yariv, 2007, "Deliberative Voting", Journal of Economic Theory, 134:317-338.

16. Gerber, Elisabeth R., Rebecca B. Morton, and Thomas A. Rietz. 1998. "Minority Representation in Multimember Districts." American Political Science Review 92:127-144.

17. Guinier, Lani. 1994. The Tyranny of the Majority. New York: Free Press.

18. Hortala-Vallve, Rafael. 2006. "Qualitative Voting." mimeo, London School of Economics.

19. Issacharoff, Samuel, Pamela Karlan, and Richard Pildes. 2002. The Law of Democracy: Legal Structure and the Political Process. 2nd ed. Foundation Press.

20. Jackson, Matthew, and Hugo Sonnenschein. 2007. "Overcoming incentive Constraints by Linking Decisions." Econometrica 75:241-57.

21. McKelvey, Richard, 1976, "Intransitivities in multidimensional voting models and some implications for agenda control", Journal of Economic Theory 12:472-482.

22. Ordeshook, Peter C. and Thomas R. Palfrey, 1988, "Agendas, Strategic Voting, and Signaling with Incomplete Information", American Journal of Political Science, 32:441-466.

23. Palfrey, Thomas R. and Howard Rosenthal, 1991, "Testing for Effects of Cheap Talk in a Public Goods Game with Private Information", Games and Economic Behavior, 3:183-220.

24. Pildes, Richard H. and Kristen A. Donoghue,1995, "Cumulative Voting in the United States", The University of Chicago Legal Forum, 1995:241-313.

25. Sopher, Barry and Inigo Zapater, 1993, "Communication and Coordination in Signalling Games: An Experimental Study", C.V.Starr Center for Applied Economics, NYU, RR \# 93-26. 


\section{Appendix A}

Proof of Proposition 1. (1) To verify that the strategies described are an equilibrium, consider $i$ 's problem at any time $t$, where $i \in \Lambda_{t}$. Voter $i$ must decide whether to cast the bonus vote on $P_{t}$ or wait for a future proposal $P_{t+k}$, $k \in\{1, . ., T-t\}$. Call $l_{t}$ the number of voters other than $i$ left with the bonus vote at $t ; p_{B s}\left(p_{1 s}\right)$ the probability of obtaining the desired outcome when casting $1+B(1)$ votes on $P_{s}$, and $\pi_{s}$ the probability that a voter $r \in \Lambda_{t}$ and other than $i$ casts the bonus vote over proposal $P_{s}$. Given the symmetry of $G$, at any $s$ the probability of obtaining the desired outcome when casting the bonus vote depends exclusively on the number of other voters who also cast the bonus vote. Thus:

$$
p_{B t}^{t}=\sum_{j=0}^{l}\left(\begin{array}{c}
l_{t} \\
j
\end{array}\right)\left(\pi_{t}^{t}\right)^{j}\left(1-\pi_{t}^{t}\right)^{l_{t}-j} p_{B}(j)
$$

where the superscript $t$ indicates the time at which the probability is evaluated, and $p_{B}(j)$ is the probability of obtaining the desired outcome when $i$ and $j$ other voters cast the bonus vote and all others do not. In the candidate equilibrium $\pi_{t}^{t}=1 /(T-t)$. Thus:

$$
p_{B t}^{t}=\sum_{j=0}^{l}\left(\begin{array}{c}
l_{t} \\
j
\end{array}\right)\left(\frac{1}{T-t}\right)^{j}\left(\frac{T-t-1}{T-t}\right)^{l_{t}-j} p_{B}(j)
$$

Similarly, evaluated at $t$, the probability of obtaining the desired outcome when casting the bonus vote at $t+k$ is given by:

$$
p_{B t+k}^{t}=\sum_{j=0}^{l}\left(\begin{array}{c}
l_{t} \\
j
\end{array}\right)\left[\prod_{s=0}^{k-1}\left(1-\pi_{t+s}^{t}\right) \pi_{t+k}^{t}\right]^{j}\left[1-\prod_{s=0}^{k-1}\left(1-\pi_{t+s}^{t}\right) \pi_{t+k}^{t}\right]^{l_{t}-j} p_{B}(j)
$$

But in the candidate equilibrium $\pi_{t+s}^{t}=1 /(T-t-s)$. The probabilities in the square brackets of equation (A.2) simplify, and we obtain:

$$
p_{B t+k}^{t}=\sum_{j=0}^{l}\left(\begin{array}{l}
l_{t} \\
j
\end{array}\right)\left(\frac{1}{T-t}\right)^{j}\left(\frac{T-t-1}{T-t}\right)^{l_{t}-j} p_{B}(j)=p_{B t}^{t}
$$

Following the same logic, we can show $p_{1 t}^{t}=p_{1 t+k}^{t}$. At time $t$ voter $i$ decides whether to cast the bonus vote on $P_{t}$ or $P_{t+k}$ by evaluating the difference in expected utility from the two actions: $E u_{i}\left(P_{t}\right)-E u_{i}\left(P_{t+k}\right)=v_{t}\left(p_{B t}^{t}-p_{1 t}^{t}\right)-$ $v_{t+k}\left(p_{B t+k}^{t}-p_{1 t+k}^{t}\right)=\left(p_{B}^{t}-p_{1}^{t}\right)\left(v_{t}-v_{t+k}\right)$. Since $p_{B}^{t} \geq p_{1}^{t}$, casting the bonus vote on $P_{t}$ if $v_{t}>v_{t+k}$ is a best response response strategy for $i$ for all $k \in\{1, . ., T-t\}$ and all $t$ : the candidate equilibrium is indeed an equilibrium.

(2) To establish part (2), note first that the strategy described in the lemma is a best response for all other voters' strategies that result in $p_{B s}^{t}=p_{B}^{t}$ and $p_{1 s}^{t}=p_{1}^{t}$ for all $s$. But with iid valuations, all strategies that depend exclusively 
on relative valuations have this property. Thus no strategy of the type: cast the bonus vote on the $k$ th higher valuation with probability $\pi_{k}$ can be an equilibrium unless $k=1$ and $\pi_{k}=1$. If another equilibrium exists, it must involve strategies where voters differentiate among the proposals on the basis of their order. For our purposes it is sufficient to show that no such equilibrium exists for specific values of $n, T$, and $B$. Suppose for example $n=3$ and $B=1$, and consider a candidate equilibrium where all voters but $i$ cast the bonus vote on some proposal $P_{r}$ with probability $\pi_{r}^{1}=q_{r}$. With no loss of generality, suppose $q_{r}>$ $1 / T$. There must then exist at least one proposal $P_{s}$ for which $\pi_{s}^{1}=q_{s}<1 / T$. With $n=3$ and $B=1, p_{1 t}^{1}=3 / 4-\left[q_{t}\left(1-q_{t}\right)\right] / 4$, and $p_{B t}^{1}=3 / 4+\left[\left(1-q_{t}\right)^{2}\right] / 8$. Voter $i$ 's best response is to cast the bonus vote on $P_{r}$ over $P_{t}$ if $v_{r} / v_{t}>\left(p_{B t}^{1}-\right.$ $\left.p_{1 t}^{1}\right) /\left(p_{B r}^{1}-p_{1 r}^{1}\right)$ for all $t$; and similarly on $P_{s}$ over $P_{t}$ if $v_{s} / v_{t}>\left(p_{B t}^{1}-p_{1 t}^{1}\right) /($ $\left.p_{B s}^{1}-p_{1 s}^{1}\right)$ for all $t$; It is easy to verify that if $q_{r}>q_{s},\left(p_{B r}^{1}-p_{1 r}^{1}\right)<\left(p_{B s}^{1}-\right.$ $\left.p_{1 s}^{1}\right)$, and thus if voter $i$ plays his best response strategy, he must be expected to cast the bonus vote on $P_{r}$ with some probability $\pi_{r i}^{1}<\pi_{s i}^{1}$ : no symmetrical equilibrium exists for $q \neq 1 / T$. Symmetrical equilibria where voters differentiate among the proposals on the basis of their order do exist for different values of $n, T$, and $B$. For example, as stated in footnote 2 , for all $n$ odd and $B<1$, there is an equilibrium where every voter casts the bonus vote on proposal $P_{t}$, $\forall t \in\{1, \ldots, T\}$.

Proof of Proposition 2. The probabilities $p_{B}$ and $p_{1}$ in (1) depend on the value of the bonus vote. The proof proceeds by verifying the proposition at the smallest value of the bonus vote for which the voting mechanism differs from simple majority voting, and thus $p_{B} \neq p \neq p_{1}$.

(1) Consider first the case of $n$ even. Any positive value of the bonus vote, no matter how small, affects the outcome with positive probability: there is always a positive probability that the voters are exactly split and that one more voter on one side than on the other casts the bonus vote. Consider then a value of the bonus vote small enough that as long as the two groups are not equally split, the bonus votes cannot modify the outcome. This threshold is defined by the condition that a minority of size $n / 2-1$ casting all bonus votes does not win over a majority of size $n / 2+1$ casting no bonus votes, or:

$$
\left(\frac{n}{2}-1\right)(1+B)<\left(\frac{n}{2}+1\right) \Longleftrightarrow B<\frac{4}{n-2}
$$

We can now derive $p_{B}$ and $p_{1}$ for all values of $B \in(0,4 /(n-2))$. The only difference with respect to simple majority voting can arise when the voters are split into groups of equal size. In this case, simple majority yields a tie, but the existence of the bonus vote creates many possible configurations of voting choices for which one side strictly wins. Consider the problem from the point of view of voter $i$. It turns out to be simpler to isolate a second voter $j$ on the opposite side of $i$. Whenever voter $j$ casts the same number of votes as $i$, the probability that $i$ 's side wins equals the probability that $j$ 's side wins, and the expected deviations from simple majority cancel out. The relevant cases are those where $i$ and $j$ cast a different number of votes. In calculating $p_{B}$ then, 
the deviation from $p$ occurs when $j$ does not cast the bonus vote. Call $M_{i}$ the number of voters on $i$ 's side who vote $1+B$, ignoring $i$, and similarly $M_{j}$ the number of voters on $j$ 's side who vote $1+B$, ignoring $j$. It is immediate that $i$ 's side wins if $M_{i}>M_{j}-1$. Similarly, $i$ 's side ties if $M_{i}=M_{j}-1$ and loses if $M_{i}<M_{j}-1$. Thus:

$p_{B}=p+\left(\frac{1}{2}\right)^{n-1}\left(\begin{array}{c}n-1 \\ n / 2\end{array}\right)\left(\frac{T-1}{T}\right)\left[\frac{1}{2} \operatorname{prob}\left(M_{i}>M_{j}-1\right)-\frac{1}{2} \operatorname{prob}\left(M_{i}<M_{j}-1\right)\right]$

where $(T-1) / T$ is the probability that voter $j$ casts a single vote, and the probabilities inside the square brackets are multiplied by $(1 / 2)$ because, absent the bonus vote, the relevant events would result in a tie. But prob $\left(M_{i}>M_{j}-\right.$ $1)=\operatorname{prob}\left(M_{i}=M_{j}\right)+\operatorname{prob}\left(M_{i}>M_{j}\right)$, and $\operatorname{prob}\left(M_{i}<M_{j}-1\right)=\operatorname{prob}\left(M_{i}<\right.$ $\left.M_{j}\right)-\operatorname{prob}\left(M_{i}=M_{j}-1\right)$. And since $\operatorname{prob}\left(M_{i}>M_{j}\right)=\operatorname{prob}\left(M_{i}<M_{j}\right)$, we can write:

$$
p_{B}=p+\left(\frac{1}{2}\right)^{n}\left(\begin{array}{c}
n-1 \\
n / 2
\end{array}\right)\left(\frac{T-1}{T}\right)\left[\operatorname{prob}\left(M_{i}=M_{j}\right)+\operatorname{prob}\left(M_{i}=M_{j}-1\right)\right]
$$

Similarly,

$$
p_{1}=p-\left(\frac{1}{2}\right)^{n}\left(\begin{array}{c}
n-1 \\
n / 2
\end{array}\right)\left(\frac{1}{T}\right)\left[\operatorname{prob}\left(M_{i}=M_{j}\right)+\operatorname{prob}\left(M_{i}=M_{j}+1\right)\right]
$$

The problem is symmetrical, and thus $\operatorname{prob}\left(M_{i}=M_{j}-1\right)=\operatorname{prob}\left(M_{j}=M_{i}-1\right)$ : the expressions in square brackets in (A.4) and (A.5) are identical. Call them $C$. We can now write:

$$
\begin{aligned}
p_{B}-p_{1} & =\left(\frac{1}{2}\right)^{n}\left(\begin{array}{c}
n-1 \\
n / 2
\end{array}\right) C \\
p-p_{1} & =\left(\frac{1}{2}\right)^{n}\left(\begin{array}{c}
n-1 \\
n / 2
\end{array}\right)\left(\frac{1}{T}\right) C
\end{aligned}
$$

We know from (1) that:

$$
E V>E W \Longleftrightarrow E v_{(T)}\left(p_{B}-p_{1}\right)>T E v\left(p-p_{1}\right)
$$

Substituting (A.6) and (A.7), we obtain:

$$
E V>E W \Longleftrightarrow E v_{(T)}>T E v\left(\frac{1}{T}\right) \text { or } E v_{(T)}>E v
$$

which is always satisfied. We can conclude that if $n$ is even, ex ante efficiency strictly improves over simple majority voting for all values of $B \in(0,4 /(n-2))$. Note that if $n=2$, the value of the bonus vote is unconstrained. 
(2) Consider now the case of $n$ odd. With $n$ odd, there are strictly positive values of the bonus vote that never alter the voting outcome from what it would be with simple majority. These are values such that when the voters are divided into two groups with opposite preferences of sizes as similar as possible $((n-1) / 2$ on one side, and $(n-1) / 2+1$ on the other), the minority loses even when all voters on the minority side cast their bonus vote, and none of the voters on the majority side does so. Or:

$$
\left(\frac{n-1}{2}\right)(1+B)<\left(\frac{n-1}{2}+1\right) \Leftrightarrow B<\frac{2}{n-1}
$$

For all $B<2 /(n-1)$ and $n$ odd, then trivially $E V \equiv E W .^{24}$ Suppose then $B \geq 2 /(n-1)$, but suppose also that the bonus vote is small enough not to matter in any other possible configuration of votes. The next smallest threshold is given by a value of $B$ such that when the two groups with opposite preferences are of sizes $(n-1) / 2$, and $(n-1) / 2+1$ and none of the majority voters uses the bonus vote while all but one of the minority voters do so, the result is a tie. I.e.:

$$
\left(\frac{n-1}{2}\right)(1+B)-B=\frac{n+1}{2} \Leftrightarrow B=\frac{2}{n-3}
$$

Suppose then $B \in[2 /(n-1), 2 /(n-3))$. From the point of view of voter $i$, when casting the bonus vote the probability of obtaining the desired outcome differs from the simple majority case only if he finds himself in a minority of size $(n-1) / 2$ and all of the voters on his side cast the bonus vote, while none of the majority voters do so. Or:

$$
p_{B}=p+\left(\frac{1}{2}\right)^{n-1+I_{\text {tie }}}\left(\begin{array}{c}
n-1 \\
(n+1) / 2
\end{array}\right)\left(\frac{1}{T}\right)^{(n-3) / 2}\left(\frac{T-1}{T}\right)^{(n+1) / 2}
$$

where $I_{\text {tie }}=1$ if $B=2 /(n-1)$, and 0 if $B \in(2 /(n-1), 2 /(n-3))$.

Similarly, when casting a single vote the difference with respect to simple majority comes from the possibility of losing, even when belonging to the majority, if all minority voters cast the bonus vote and none of the majority voters do so:

$$
p_{1}=p-\left(\frac{1}{2}\right)^{n-1+I_{\text {tie }}}\left(\begin{array}{c}
n-1 \\
(n-1) / 2
\end{array}\right)\left(\frac{1}{T}\right)^{(n-1) / 2}\left(\frac{T-1}{T}\right)^{(n-1) / 2}
$$

where the difference in the binomial term reflects the different size of the two groups and the fact that $p_{B}$ in (A.9) is calculated supposing that voter $i$ is in the minority group, and $p_{1}$ in (A.10) supposing $i$ is in the majority. Hence:

$p_{B}-p_{1}=\left(\frac{1}{2}\right)^{n-1+I_{\text {tie }}}\left(\frac{1}{T}\right)^{(n-1) / 2}\left(\frac{T-1}{T}\right)^{(n-1) / 2}\left[\left(\begin{array}{c}n-1 \\ (n+1) / 2\end{array}\right)(T-1)+\left(\begin{array}{c}n-1 \\ (n-1) / 2\end{array}\right)\right]$

\footnotetext{
${ }^{24}$ A simple example makes the point clearly. Suppose $n=3$. Then for all $B<1$, a voter can win only if at least one of the other two voters agrees with him, exactly as in the case of simple majority voting
} 
Substituting (A.10) and (A.11) in (A.8):

$$
E V>E W \Longleftrightarrow D\left[E v_{(T)}\left(\frac{T(n-1)+2}{n+1}\right)-T E v\right]>0
$$

where:

$$
D \equiv\left(\frac{1}{2}\right)^{n-1+I_{t i e}}\left(\frac{1}{T}\right)^{(n-1) / 2}\left(\frac{T-1}{T}\right)^{(n-1) / 2}\left(\begin{array}{c}
n-1 \\
(n-1) / 2
\end{array}\right)>0
$$

Or:

$$
E V-E W>0 \Longleftrightarrow \frac{E v_{(T)}}{E v}>\frac{T(n+1)}{T(n-1)+2}
$$

This is the condition in Proposition 2

\section{Construction of Figure 1.}

(a) Derivation of $p_{1}, p_{B}$ for arbitrary $B$.

Consider the problem from the point of view of voter $i$. The expected voting behavior of the other voters is identical over all proposals. Consider then a proposal $P$, and call $\pi$ the ex ante probability that any voter casts the bonus vote on that proposal (where in equilibrium $\pi=1 / T$ ). Call $J$ the number of other voters who cast the bonus vote. Excluding $i$, the total number of votes cast in the election is $n-1+B J$. Call $M_{i}\left(m_{i}\right)$ the number of voters on $i$ 's side who vote $1+B(1)$ over $P$, and thus $m_{i}+(1+B) M_{i}$ the total number of votes on $i$ 's side, ignoring $i$, and $n-1+B J-m_{i}-(1+B) M_{i}$ the total number of votes on the opposite side. If voter $i$ casts only his regular vote, for any $M_{i}$ and $J$, voter $i$ 's side wins strictly if: $m_{i}+(1+B) M_{i}+1>n-1+B J-m_{i}-(1+B) M_{i}$, or $m_{i}>\left[n+B\left(J-2 M_{i}\right)\right] / 2-1-M_{i}$; and ties if $m_{i}=\left[n+B\left(J-2 M_{i}\right)\right] / 2-1-M_{i}$. Taking into account that for generic $n, J$, and $B\left[n+B\left(J-2 M_{i}\right)\right] / 2$ need not be an integer, we can write:

$p_{1}=\sum_{J=0}^{n-1}\left(\begin{array}{c}n-1 \\ J\end{array}\right) \sum_{M_{i}=0}^{J}\left(\begin{array}{c}J \\ M_{i}\end{array}\right)\left[\sum_{m_{i}=k}^{n-1-J}\left(\begin{array}{c}n-1-J \\ m_{i}\end{array}\right)+\frac{I_{I}}{2}\left(\begin{array}{c}n-1-J \\ \widetilde{k}\end{array}\right)\right] \pi^{J}(1-\pi)^{n-1-J}(1 / 2)^{n-1}$

with:

$$
k \equiv I\left[\frac{n+B\left(J-2 M_{i}\right)}{2}\right]-M_{i}
$$

where $I[x]$ is the largest integer smaller than $x$;

$$
\begin{gathered}
\widetilde{k} \equiv \frac{n+B\left(J-2 M_{i}\right)}{2}-1-M_{i}, \\
I_{I}=\left\{\begin{array}{c}
1 \text { if } \widetilde{k} \text { is an integer } \\
0 \text { otherwise }
\end{array}\right.
\end{gathered}
$$

If voter $i$ casts his bonus vote, then for any $M_{i}$ and $J$, voter $i$ 's side wins strictly if: $m_{i}+(1+B) M_{i}+1+B>n-1+B J-m_{i}-(1+B) M_{i}$, or $m_{i}>$ 
$\left[n+B\left(J-1-2 M_{i}\right)\right] / 2-1-M_{i}$; and ties if $m_{i}=\left[n+B\left(J-1-2 M_{i}\right)\right] / 2-1-M_{i}$.

We can write:

$p_{B}=\sum_{J=0}^{n-1}\left(\begin{array}{c}n-1 \\ J\end{array}\right) \sum_{M_{i}=0}^{J}\left(\begin{array}{c}J \\ M_{i}\end{array}\right)\left[\sum_{m_{i}=k_{B}}^{n-1-J}\left(\begin{array}{c}n-1-J \\ m_{i}\end{array}\right)+\frac{I_{I}}{2}\left(\begin{array}{c}n-1-J \\ \widetilde{k_{B}}\end{array}\right)\right] \pi^{J}(1-\pi)^{n-1-J}(1 / 2)^{n-1}$

with:

$$
k_{B} \equiv I\left[\frac{n+B\left(J-1-2 M_{i}\right)}{2}\right]-M_{i}
$$

where $I[x]$ is the largest integer smaller than $x$;

$$
\begin{gathered}
\widetilde{k}_{B} \equiv \frac{n+B\left(J-1-2 M_{i}\right)}{2}-1-M_{i} ; \\
I_{I}=\left\{\begin{array}{c}
1 \text { if } \widetilde{k}_{B} \text { is an integer } \\
0 \text { otherwise }
\end{array}\right.
\end{gathered}
$$

\section{(b) Efficiency}

Expected first best efficiency is given by:

$E V^{*}=(1 / 2)^{n-1}\left[1 / 2+\sum_{k=0}^{n-2}\left(\begin{array}{c}n-1 \\ k\end{array}\right)\left(\int_{0}^{k}(1 / 2) f(x) d x+\int_{k}^{k+1} \int_{x-k}^{1} \operatorname{sdsf}(x) d x\right)\right]$

where $f$ is the density function of a sum of $n-1$ independent variables. When the $n-1$ variables are each distributed according to a Uniform over the interval $[0,1]$ :

$$
f(x)=\sum_{k=0}^{n}(-1)_{k}\left(\begin{array}{c}
n-1 \\
k
\end{array}\right)(x-k)^{n-2} \operatorname{Sign}[x-k] \frac{1}{2(n-2) !}
$$

Proof of Proposition 4. I begin by studying the first candidate equilibrium. Recall that each voter $i$, including the chair, decides whether to cast the bonus vote on $P_{1}$ or $P_{2}$ by evaluating the difference in expected utility from the two actions: $E u_{i}\left(P_{1}\right)-E u_{i}\left(P_{2}\right)=v_{i 1}\left(p_{i B 1}-p_{i 11}\right)-v_{i 2}\left(p_{i B 2}-p_{i 12}\right)$, where all probabilities are evaluated at the time of the first decision. Any voter's best response strategy is to cast the bonus vote on $P_{1}$ if and only if:

$$
\frac{v_{i 1}}{v_{i 2}} \geq \frac{p_{i B 2}-p_{i 12}}{p_{i B 1}-p_{i 11}}
$$

where $i=c$ if the voter is the chair, and I ignore the subscript otherwise.

Restricting the value of the bonus vote to $B \in \mathcal{B}(n)$ allows us to calculate (A.12) and establish its properties.

(1) Suppose first that $n$ is even. The derivation of the relevant probabilities proceeds as in the proof of Proposition 2. Consider first the problem for a voter $i$ who is not the chair, and begin by deriving $p_{B 1}$, the probability of winning $P_{1}$ when casting the bonus vote. Recall that $B<4 /(n-2)$, implying that 
the bonus vote can make a difference only if the two groups on opposite side of any proposal are of equal size. With probability $1 / 2$, the chair is against $i$, the chair's bonus vote cancels $i$ 's, and $p_{B 1}=p$ (where $p$ is the probability of winning $P_{1}$ under simple majority). With probability $1 / 2$, the chair is on $i$ 's side. In this case, consider two voters on the opposite side, one of whom I call $j$. If both cast their bonus votes, again $p_{B 1}=p$. However, if either one or both do not cast their bonus vote, then $p_{B 1}>p$. Working through the different scenarios, we can derive:

$$
p_{B 1}=p+\left(\frac{1}{2}\right)^{n-1}\left(\begin{array}{c}
n-2 \\
n / 2
\end{array}\right)\left[\left(1-\pi_{1}\right) A_{11}+\frac{\left(1+\pi_{1}\right)}{2} A_{01}+\frac{\left(1-\pi_{1}\right)}{2} A_{21}\right]
$$

where $A_{0 t}, A_{1 t}$, and $A_{2 t}$ are defined as:

$$
\begin{aligned}
& A_{0 t} \equiv \operatorname{prob}\left(M_{i t}=M_{j t}\right)=\sum_{M=0}^{n / 2-2}\left(\begin{array}{c}
n / 2-2 \\
M
\end{array}\right)\left(\begin{array}{c}
n / 2-2 \\
M
\end{array}\right) \pi_{t}^{2 M}\left(1-\pi_{t}\right)^{n-4-2 M} \\
& A_{1 t} \equiv \operatorname{prob}\left(M_{i t}=M_{j t}+1\right)=\sum_{M=0}^{n / 2-3}\left(\begin{array}{c}
n / 2-2 \\
M
\end{array}\right)\left(\begin{array}{c}
n / 2-2 \\
M+1
\end{array}\right) \pi_{t}^{2 M+1}\left(1-\pi_{t}\right)^{n-5-2 M} \\
& A_{2 t} \equiv \operatorname{prob}\left(M_{i t}=M_{j t}+2\right)=\sum_{M=0}^{n / 2-4}\left(\begin{array}{c}
n / 2-2 \\
M
\end{array}\right)\left(\begin{array}{c}
n / 2-2 \\
M+2
\end{array}\right) \pi_{t}^{2 M+2}\left(1-\pi_{t}\right)^{n-6-2 M},
\end{aligned}
$$

and $M_{i t}\left(M_{j t}\right)$ is defined as the number of voters on $i$ 's $(j$ 's) side, up to a maximum of $n / 2-2$ who cast the bonus vote on $P_{t}$. The maximum is $n / 2-2$ because we always exclude voter $i$ and account separately for the chair, and, to keep the two sides balanced, for $j$ and one other voter on $j$ 's side. The details are cumbersome but not difficult, and we obtain ${ }^{25}$ :

$$
\begin{aligned}
p_{B 1}-p_{11}= & \left(\frac{1}{2}\right)^{n-1}\left\{\left(\begin{array}{c}
n-2 \\
n / 2
\end{array}\right)\left[\left(A_{11}+A_{01}\right)+\left(A_{21}-A_{01}\right)\left(1-\pi_{1}\right)^{2} \mathrm{~A} . \mathrm{A3}\right)\right. \\
& \left.+\left(\begin{array}{c}
n-2 \\
n / 2-1
\end{array}\right)\left[\left(A_{11}+A_{01}\right)+\left(A_{21}-A_{01}\right) \pi_{1}\left(1-\pi_{1}\right)\right]\right\}
\end{aligned}
$$

When deriving $p_{B 2}-p_{12}$, on the other hand, voter $i$ knows that the chair will not cast the bonus vote, and thus the expression will not be exactly symmetrical to (A.13). Again the details are somewhat cumbersome, but the logic straightforward. The expression is:

$$
\begin{aligned}
p_{B 2}-p_{12}= & \left(\frac{1}{2}\right)^{n-1}\left\{\left(\begin{array}{c}
n-2 \\
n / 2
\end{array}\right)\left[\left(A_{12}+A_{02}\right)+\left(A_{22}-A_{02}\right) \pi_{2}^{2}\right]\right. \\
& \left.+\left(\begin{array}{c}
n-2 \\
n / 2-1
\end{array}\right)\left[\left(A_{12}+A_{02}\right)+\left(A_{22}-A_{02}\right) \pi_{2}\left(1-\pi_{2}\right)\right]\right\}
\end{aligned}
$$

With $T=2, \pi_{1}=1-\pi_{2}$, and simple manipulations show that for all $\pi_{1}$, $A_{11}=A_{12} ; A_{01}=A_{02}$, and $A_{21}=A_{22}$. It follows that $p_{B 1}-p_{11}=p_{B 2}-p_{12}$.

\footnotetext{
${ }^{25}$ The detailed derivation is available upon request.
} 
A voter $i$ who is not the chair should cast the bonus vote on $P_{1}$ if and only if $v_{i 1} \geq v_{i 2}$. Hence $\alpha(n, G)=1$, and $\pi_{1}=1 / 2$.

Consider now the chair's problem. Given $\pi_{1}=1 / 2=\pi_{2}$, the chair's best response is simply to cast his bonus vote on the proposal to which he attaches highest valuation. Thus if $v_{c 1} \geq v_{c 2}$, always casting the bonus vote on $P_{1}$ is indeed supported as an equilibrium strategy. But we also know from Proposition 3 that with $\pi_{1}=1 / 2=\pi_{2}$ from the point of view of the chair the order of proposals is irrelevant, and choosing $P_{1}$ such that $v_{c 1} \geq v_{c 2}$ is consistent with the chair's best response.

We can conclude that if $n$ is even and $T=2$, there is indeed an equilibrium where $v_{c 1} \geq v_{c 2}, x_{c 1}=1+B$, and $x_{1}=1+B$ if and only if $v_{1} \geq v_{2}$, or equivalently $\alpha(n, G)=1$ for all $G$. The equilibrium is fully informative because it is common knowledge that the chair always orders his higher priority proposal first.

(2) Suppose now that $n$ is odd. Again consider first the problem for a voter $i$ who is not the chair. Recall that $B \in \mathcal{B}(n)=[2 /(n-1), 2 /(n-3))$, implying that the bonus vote makes a difference over simple majority only when the groups on opposite sides of a proposal differ in size by a single voter; all minority voters cast the bonus vote, and none of the majority does. In the candidate equilibrium, the chair always casts his bonus vote on $P_{1}$. Thus $p_{B 1} \neq p$ only if the chair is on $i^{\prime} s$ side and so are $(n-1) / 2-2$ other voters, all of which cast their bonus vote, while none of the others do. That is:

$$
p_{B 1}=p+\left(\frac{1}{2}\right)^{n-1+I_{\text {tie }}}\left(\begin{array}{c}
n-2 \\
(n+1) / 2
\end{array}\right) \pi_{1}^{(n-5) / 2}\left(1-\pi_{1}\right)^{(n+1) / 2}
$$

The same logic allows us to derive $p_{11}, p_{B 2}$, and $p_{12}$, and we obtain:

$p_{B 1}-p_{11}=\left(\frac{1}{2}\right)^{n-1+I_{\text {tie }}} \pi_{1}^{(n-5) / 2}\left(1-\pi_{1}\right)^{(n-1) / 2}\left[\left(\begin{array}{c}n-2 \\ (n+1) / 2\end{array}\right)\left(1-\pi_{1}\right)+\left(\begin{array}{c}n-2 \\ (n-1) / 2\end{array}\right) \pi_{1}\right]$

and:

$$
p_{B 2}-p_{12}=\left(\frac{1}{2}\right)^{n-1+I_{t i e}}\left(\begin{array}{c}
n-2 \\
(n-1) / 2
\end{array}\right)\left[\pi_{2}\left(1-\pi_{2}\right)\right]^{(n-3) / 2}
$$

In the candidate equilibrium $\pi_{1} \in(0,1)$ and the ratio of (A.17) to (A.16) is well defined. Expanding the binomial terms and simplifying, we can write:

$$
\frac{p_{B 2}-p_{12}}{p_{B 1}-p_{11}}=\left[\frac{\pi_{2}\left(1-\pi_{2}\right)}{\pi_{1}\left(1-\pi_{1}\right)}\right]^{(n-3) / 2}\left(\frac{\pi_{1}}{1-\pi_{1}}\right)\left(\frac{n+1}{n-3+4 \pi_{1}}\right)
$$

Taking into account that $\pi_{1}=1-\pi_{2}$, (A.18) simplifies to:

$$
\frac{p_{B 2}-p_{12}}{p_{B 1}-p_{11}}=\left(\frac{\pi_{1}}{1-\pi_{1}}\right)\left(\frac{n+1}{n-3+4 \pi_{1}}\right) \equiv \beta\left(\pi_{1}\right)
$$

Voter $i$ 's best response strategy is to cast the bonus vote on $P_{1}$ if $v_{i 1} \geq$ $\beta\left(\pi_{1}\right) v_{i 2}$. Given $\pi_{c 1}=1$, a symmetrical best response by all voters who are not 
the chair amounts to finding a value of $\pi_{1}$ that solves $\pi_{1}=\operatorname{prob}\left(v_{1} \geq \beta\left(\pi_{1}\right) v_{2}\right)$, or:

$$
\pi_{1}=\int_{0}^{1} F\left(\left(\frac{v}{\beta\left(\pi_{1}\right)}\right) d F(v)\right.
$$

With $v_{1}$ and $v_{2}$ independent and distributed according to $F$, the strategy described in Proposition 4 is a symmetrical best response if and only if, for all $n$ and $G$, there exists a solution to (A.19) at some $\widetilde{\pi}_{1} \in(0,1 / 2]$. For given $n$ and $G$, call the right-hand side of (A.19) $\varphi\left(\pi_{1}\right)$. The function $\varphi\left(\pi_{1}\right)$ is continuous in $\pi_{1}$ and for all $n \geq 3, \partial \beta\left(\pi_{1}\right) / \partial \pi_{1}>0$, implying that $\partial \varphi\left(\pi_{1}\right) / \partial \pi_{1} \leq 0$. But $\lim _{\pi_{1} \rightarrow 0} \beta\left(\pi_{1}\right)=0$, and hence $\lim _{\pi_{1} \rightarrow 0} \varphi\left(\pi_{1}\right)=1$, while $\beta(1 / 2)=(n+1) /(n-$ 1) $>1$ for all finite $n$, and hence $\varphi(1 / 2)<1 / 2$. Thus there exists a unique $\widetilde{\pi}_{1} \in(0,1 / 2)$ such that $\widetilde{\pi}_{1}=\varphi\left(\widetilde{\pi}_{1}\right)$, or equivalently a unique $\alpha(n, G)>1$ : the statement in the Proposition is verified.

Consider now the chair's problem. The logic is unchanged. From the chair's point of view, $p_{c B t} \neq p$ only if the chair is in a minority of size $(n-1) / 2$ all of whose members cast their bonus vote, while none of the others do. That is:

$$
p_{c B t}=p+\left(\frac{1}{2}\right)^{n-1+I_{t i e}}\left(\begin{array}{c}
n-1 \\
(n+1) / 2
\end{array}\right) \pi_{t}^{(n-3) / 2}\left(1-\pi_{t}\right)^{(n+1) / 2} \quad t=1,2 .
$$

Similarly, $p_{c 1 t} \neq p$ only if the chair is in a majority of size $(n+1) / 2$ none of whose members cast their bonus vote, while all of the others do:

$$
p_{c 1 t}=p-\left(\frac{1}{2}\right)^{n-1+I_{t i e}}\left(\begin{array}{c}
n-1 \\
(n-1) / 2
\end{array}\right) \pi_{t}^{(n-1) / 2}\left(1-\pi_{t}\right)^{(n-1) / 2} \quad t=1,2 .
$$

Thus:

$p_{c B t}-p_{c 1 t}=\left(\frac{1}{2}\right)^{n-1+I_{t i e}} \pi_{t}^{(n-1) / 2}\left(1-\pi_{t}\right)^{(n-1) / 2}\left[\left(\begin{array}{c}n-1 \\ (n+1) / 2\end{array}\right)\left(\frac{1-\pi_{t}}{\pi_{t}}\right)+\left(\begin{array}{c}n-1 \\ (n-1) / 2\end{array}\right)\right]$

with $t=1,2$. If $\pi_{1} \in(0,1)$ we can write:

$$
\frac{p_{c B 2}-p_{c 12}}{p_{c B 1}-p_{c 11}}=\left[\frac{\pi_{2}\left(1-\pi_{2}\right)}{\pi_{1}\left(1-\pi_{1}\right)}\right]^{(n-1) / 2}\left(\frac{\pi_{1}}{\pi_{2}}\right)\left(\frac{n-1+2 \pi_{2}}{n-1+2 \pi_{1}}\right)
$$

or, again taking into account that $\pi_{1}=1-\pi_{2}$ and simplifying:

$$
\frac{p_{c B 2}-p_{c 12}}{p_{c B 1}-p_{c 11}}=\left(\frac{\pi_{1}}{1-\pi_{1}}\right)\left(\frac{n+1-2 \pi_{1}}{n-1+2 \pi_{1}}\right)
$$

The right-hand side of (A.22) is strictly increasing in $\pi_{1}$, and equals 1 at $\pi_{1}=1 / 2$; thus $\left(p_{c B 2}-p_{c 12}\right) /\left(p_{c B 1}-p_{c 11}\right)<1$ for all $\pi_{1}<1 / 2$. It follows that if $v_{c 1} \geq v_{c 2}, x_{c 1}=1+B$ is indeed the chair's best response strategy.

Finally, given $\pi_{1}<1 / 2$, could the chair gain by changing the order of proposals? Two deviations need to be ruled out. The chair could choose $P_{1}$ such that $v_{c 1}<v_{c 2}$ and (i) cast the bonus vote on $P_{2}$, or (ii) still cast the bonus 
vote on $P_{1}$. Call $v_{c}^{*} \equiv \max \left\{v_{c 1}, v_{c 2}\right\}$ and $v_{c} \equiv \min \left\{v_{c 1}, v_{c 2}\right\}$. The first deviation is ruled out if: $p_{c B 1} v_{c}^{*}+p_{c 12} \underline{v_{c}} \geq p_{c 11} \underline{v_{c}}+p_{c B 2} v_{c}^{*}$, or $v_{c}^{*}\left(p_{c B 1}-p_{c B 2}\right) \geq \underline{v_{c}}($ $\left.p_{c 11}-p_{c 12}\right)$. Given $v_{c}^{*} \geq v_{c}$ and $p_{c B 1}>p_{c 11}, p_{c B 2}>p_{c 12}$, a sufficient condition is then $\left(p_{c B 1}-p_{c 11}\right) \geq\left(p_{c B 2}-p_{c 12}\right)$ which we know satisfied for all $\pi_{1}<1 / 2$ : the deviation cannot be profitable. The second deviation is ruled out if: $p_{c B 1} v_{c}^{*}+p_{c 12} \underline{v_{c}} \geq p_{c B 1} \underline{v_{c}}+p_{c 12} v_{c}^{*}$, or $v_{c}^{*}\left(p_{c B 1}-p_{c 12}\right) \geq \underline{v}_{c}\left(p_{c B 1}-p_{c 12}\right)$, or $p_{c B 1}-p_{c 12} \geq 0$. This can be verified immediately from (A.20) and (A.21) above: again the deviation cannot be profitable.

We can conclude that for both $n$ odd and $n$ even, the strategies described in the first half of the Proposition are indeed equilibrium strategies. But with $T=2$, the two proposals are completely symmetrical, and the proof can be applied identically to proposal 2 . The two equilibria are mirror-images of each other, and are payoff-equivalent.

(3) The proposition states that no other quasi-symmetric informative equilibria exist for all $n$ and $G$. We need to rule out partially informative equilibria where the chair sets his highest priority proposal as $P_{1}$ (or $P_{2}$ ) with some probability $\in(1 / 2,1)$. With $T=2$, such equilibria do indeed exist when $n$ is even. But suppose $n=3$. Consider candidate informative equilibria where $\pi_{c 1} \in(1 / 2,1]$. Taking into account $\pi_{2}=1-\pi_{1}$ and $\pi_{c 2}=1-\pi_{c 1}$ :

$$
\begin{aligned}
& p_{B 1}-p_{11}=\left(1-\pi_{1} \pi_{c 1}\right) / 8 \\
& p_{B 2}-p_{12}=\left(\pi_{c 1}+\pi_{1}-\pi_{1} \pi_{c 1}\right) / 8
\end{aligned}
$$

If $\pi_{c 1}+\pi_{1}>1$, then $p_{B 2}-p_{12}>p_{B 1}-p_{11}$, implying $\pi_{1}<1 / 2$. But we showed above that if $\pi_{1}<1 / 2$, the chair's best response is to choose $P_{1}$ such that $v_{c 1} \geq$ $v_{c 2}$ and always cast the bonus vote on $P_{1}$. The only symmetric best response for the other voters then is to cast the bonus vote on $P_{1}$ if and only if $v_{1} \geq \alpha(3, G) v_{2}$, an event that occurs with probability $\widetilde{\pi}_{1} \in(0,1 / 2)$ - the equilibrium strategy characterized earlier. If $\pi_{c 1}+\pi_{1} \leq 1$, then $p_{B 2}-p_{12} \leq p_{B 1}-p_{11}$, implying $\pi_{1} \geq 1 / 2$; but $\pi_{c 1}>1 / 2$, and thus $\pi_{c 1}+\pi_{1}>1$, a contradiction. Identical reasoning rules out any informative equilibrium with $\pi_{c 2} \in(1 / 2,1]$ that would differ from the second equilibrium characterized in the Proposition.

The focus of the analysis, and the definition of informative equilibria stress the possibility to transmit information on the chair's priorities. But could the order of proposals be used to transmit information about the chair's direction of preferences, as opposed to the chair's intensity of preferences? With $T=2$, could there be an equilibrium where if there is at least one proposal the chair favors, it will be ordered first, and if there is at least one proposal the chair opposes it will be ordered last? Call $d_{t}$ the probability that the chair favors $P_{t}$ - is there an equilibrium where $d_{1}>1 / 2$ and $d_{2}<1 / 2$ ? The answer is that no such equilibrium can exist for all $n$. Intuitively, transmitting information about one's priorities can encourage allies to withdraw their bonus vote, relative to voters with opposite preferences, reducing the chair's probability of winning the current proposal, and because preferences are independent across proposals and voters no future benefit can accrue to the chair from the reallocation of available bonus votes among the other voters. Suppose for example $n=3$, and call $i$ and 
$j$ non-chair voters such that $\mathrm{v}_{i 1}>0$ and $\mathrm{v}_{j 1}<0$ Then:

$$
\begin{aligned}
p_{B 1 i}-p_{11 i} & =\left[1-\pi_{1}-d_{1}\left(1-2 \pi_{1}+\pi_{1} \pi_{c 1}\right)\right] / 4 \\
p_{B 1 j}-p_{11 j} & =\left[1-\pi_{1}-\left(1-d_{1}\right)\left(1-2 \pi_{1}+\pi_{1} \pi_{c 1}\right)\right] / 4
\end{aligned}
$$

It can be verified immediately that $\left(p_{B 1 i}-p_{11 i}\right)<\left(p_{B 1 j}-p_{11 j}\right)$ iff $d_{1}>1 / 2$. Hence the threshold value of $v_{1} / v_{2}$ that induces a voter to cast the bonus vote on $P_{1}$ is higher if the voter agrees with the chair. As a result the chair would be better off by lying. The argument extends unchanged to $T>2$, and the possibility of equilibria of this type is not discussed further.

Proof of Proposition 5. For $S=0$, the equilibrium falls under Proposition 3; for $T=2$, and $S=1$, the equilibrium falls under Proposition 4 . Consider then $S>0$ and $T>2$. Call $\mathbf{P}_{\mathcal{S}}$ the set of $S$ proposals ordered by the chair in the first $S$ positions of the agenda, and $\mathbf{P}_{T-\mathcal{S}}$ the set of $T-S$ proposals ordered in the last $T-S$ positions. The proof is logically identical to the proof of Proposition 4.

(1) Suppose first $n=4$. Consider the best response strategy for voter $i$, who is not the chair. Voter $i$ casts the bonus vote on proposal $P_{t}$ over proposal $P_{s}$ if the ratio $v_{i t} / v_{i s}$ is larger than:

$$
\frac{p_{B s}-p_{1 s}}{p_{B t}-p_{1 t}}=\frac{3-\pi_{s}\left(2-\pi_{s}\right)-\pi_{c s}\left(1-2 \pi_{s}\right)}{3-\pi_{t}\left(2-\pi_{t}\right)-\pi_{c t}\left(1-2 \pi_{t}\right)}
$$

where the right-hand side of (A.23) is derived taking into account $B \in \mathcal{B}(4)$, as in the proof of Proposition 4 (but allowing $\pi_{c}$ to differ from 0 or 1). If $P_{s}$ and $P_{t}$ $\in \mathbf{P}_{\mathcal{S}}$, then in the candidate equilibrium $\pi_{c t}=\pi_{c s}=0$, and $\pi_{t}$, the probability that the other non-chair voter in the 3-person committee casts his bonus vote on $P_{t}$, must equal $\operatorname{prob}\left(v_{j t}=v_{j \mathcal{S}}^{*}\right) \operatorname{prob}\left(v_{j \mathcal{S}}^{*}>(1 / \alpha) v_{j \mathcal{T}-\mathcal{S}}^{*}\right)=(1 / S) \operatorname{prob}\left(v_{j \mathcal{S}}^{*}>\right.$ $\left.(1 / \alpha) v_{j \mathcal{T}-\mathcal{S}}^{*}\right)=\pi_{r}$. Thus the ratio on the right-hand side of (A.23) is equal to 1 , and $i$ will cast his bonus vote on $P_{t}$, as opposed to $P_{s}$, only if $v_{i t}>v_{i s}$. Among all proposals in $\mathbf{P}_{\mathcal{S}}$, the only candidate for $i$ 's bonus vote is the highest intensity proposal in the set, $P_{i \mathcal{S}}^{*}$. Similarly, if $P_{s}$ and $P_{t} \in \mathbf{P}_{\mathcal{T}-\mathcal{S}}$, then in the candidate equilibrium $\pi_{c t}=\pi_{c s}=1 /(T-S)$, and $\pi_{t}=\operatorname{prob}\left(v_{j t}=v_{j \mathcal{T}-\mathcal{S}}^{*}\right) \operatorname{prob}\left(v_{j \mathcal{T}-\mathcal{S}}^{*}>\right.$ $\left.\alpha v_{j \mathcal{S}}^{*}\right)=1 /(T-S) \operatorname{prob}\left(v_{j \mathcal{T}-\mathcal{S}}^{*}>\alpha v_{j \mathcal{S}}^{*}\right)=\pi_{s}$. Again, the ratio on the right-hand side of (A.23) equals 1 , and among all proposals in $\mathbf{P}_{\mathcal{T}-\mathcal{S}}$, the only candidate for $i$ 's bonus vote is the highest intensity proposal in the set, $P_{i \mathcal{T}-\mathcal{S}}^{*}$. Suppose then $P_{t}=P_{i \mathcal{T}-\mathcal{S}}^{*}$ and $P_{s}=P_{i \mathcal{S}}^{*}$ (note that this case is only relevant if $S>0$ ). The ratio in (A.23) becomes:

$$
\frac{p_{B s}-p_{1 s}}{p_{B t}-p_{1 t}}=\frac{3+\pi_{s}^{2}-2 \pi_{s}}{3-\pi_{t}\left(2-\pi_{t}\right)-\left(1-2 \pi_{t}\right) /(T-S)}
$$

where $\pi_{s}=(1 / S)\left[1-\pi_{t}(T-S)\right]$. We can write the right-hand side of (A.23) as some function $\gamma\left(\pi_{t}\right)$. Voter $i$ 's best response is to cast his bonus vote on $P_{i \mathcal{T}-\mathcal{S}}^{*}$ if and only if $v_{i \mathcal{T}-\mathcal{S}}^{*} \geq \gamma\left(\pi_{t}\right) v_{i \mathcal{S}}^{*}$. Thus in a quasi-symmetrical equilibrium $\pi_{t}$, 
the probability that a non-chair voter in the committee casts his bonus vote on $P_{t}$, must equal $\operatorname{prob}\left(v_{j t}=v_{j \mathcal{T}-\mathcal{S}}^{*}\right) \operatorname{prob}\left(v_{j \mathcal{T}-\mathcal{S}}^{*}>\gamma\left(\pi_{t}\right) v_{j \mathcal{S}}^{*}\right)$. Or:

$$
\pi_{t}=\int_{0}^{1}\left[F\left(\frac{v}{\gamma\left(\pi_{t}\right)}\right)\right]^{S}[F(v)]^{T-S-1} d F(v) \equiv \psi\left(\pi_{t}\right)
$$

Both $\gamma$ and $\psi$ are continuous in $\pi_{t}$. Note that $\lim _{\pi_{1 \rightarrow 0}} \gamma\left(\pi_{t}\right) \in(0,3 / 2)$ for all $S<T$, and hence $\lim _{\pi_{t \rightarrow 0}} \psi\left(\pi_{t}\right)>0$; while $\left.\gamma\left(\pi_{t}\right)\right|_{\pi_{t}=1 / T}>1$ for all $T>2$, and hence $\left.\psi\left(\pi_{t}\right)\right|_{\pi_{t}=1 / T}<1 / T$. It follows that (A.24) must have a solution at some $\widetilde{\pi_{t}}<1 / T$ where $\gamma\left(\widetilde{\pi_{t}}\right)=\alpha(4, G, T, S)>1$. In addition, for all $S<T-1, \gamma$ is strictly increasing in $\pi_{t}$, for all $\pi_{t} \in[0,1 /(T-S)]$, and thus $\psi$ must be strictly decreasing, and $\widetilde{\pi_{t}}$ must be unique. If $S=T-1, \lim _{\pi_{t \rightarrow 1 /(T-S)}} \gamma\left(\pi_{t}\right)=1$, and hence $\lim _{\pi_{1 \rightarrow 1 /(T-S)}} \psi\left(\pi_{t}\right)=1 / T$; but $\partial \gamma / \partial \pi_{t}$ has a unique root, a maximum, at some $\pi_{t}^{\prime}<1 / T$ (and thus $\psi$ has a unique minimum at $\pi_{t}^{\prime}$ ), implying that equation (A.24) again has a unique solution: $\alpha(4, G, T, S)$ is unique. Finally, notice that for all $\gamma\left(\widetilde{\pi_{t}}\right)>1, \psi\left(\widetilde{\pi_{t}}\right)$ must be lower the higher is $S$, and thus for given $G$ and $T, \widetilde{\pi_{t}}$ must be lower the higher is $S$. The highest admissible value of $S$ is $S=T-1$ or $T-S=1$ - the fully informative equilibrium: $\widetilde{\pi}_{t}$ is monotonically lower the more informative is the equilibrium.

Consider now the chair's problem. Given the order of the proposals and the other voters' posited strategies, the only possible targets for the chair's bonus vote are his highest intensity proposals in the two sets, $P_{c \mathcal{T}-\mathcal{S}}^{*}$ and $P_{c \mathcal{S}}^{*}$ (because in all comparisons between proposals $P_{s}$ and $P_{t}$ in the same set, whether $\mathbf{P}_{\mathcal{S}}$ or $\mathbf{P}_{\mathcal{T}-\mathcal{S}}, \pi_{t}=\pi_{s}$ and thus $\left.\left(p_{c B s}-p_{c 1 s}\right) /\left(p_{c B t}-p_{c 1 t}\right)=1\right)$. Suppose then $P_{t}=$ $P_{i \mathcal{T}-\mathcal{S}}^{*}$ and $P_{s}=P_{i \mathcal{S}}^{*}$, and recall that in the candidate equilibrium $v_{c \mathcal{T}-\mathcal{S}}^{*} \geq v_{c \mathcal{S}}^{*}$. With $B \in \mathcal{B}(4)$ and $n=4$ :

$$
\frac{p_{c B s}-p_{c 1 s}}{p_{c B t}-p_{c 1 t}}=\left(\frac{1-\pi_{s}\left(1-\pi_{s}\right)}{1-\pi_{t}\left(1-\pi_{t}\right)}\right)
$$

an expression smaller than 1 for all $\pi_{s}>\pi_{t}$. Thus if $v_{c \mathcal{T}-\mathcal{S}}^{*} \geq v_{c \mathcal{S}}^{*}$, it is indeed optimal for the chair to cast his bonus vote on $P_{i \mathcal{T}-\mathcal{S}}^{*}$. Can the chair do better by deviating in the order of the proposals? As in the proof of Proposition 4, two possible deviations must be considered. First, the chair could order his highest intensity proposal in the first $S$ positions on the agenda, and cast on it his bonus vote. Second, the chair could order his highest intensity proposal in the first $S$ positions, but cast his bonus vote on $P_{c \mathcal{T}-\mathcal{S}}^{*}$. Using the index $t$ to refer to a proposal in $\mathbf{P}_{\mathcal{T}-\mathcal{S}}$ and the index $s$ to refer to a proposal in $\mathbf{P}_{\mathcal{S}}$, the first deviation is ruled out if $v_{c}^{*}\left(p_{c B t}-p_{c B s}\right)>v_{c}\left(p_{c 1 t}-p_{c 1 s}\right)$ for all $v_{c} \leq v_{c}^{*}$. A sufficient condition then is $\left(p_{c B t}-p_{c 1 t}\right)>\left(p_{c B s}-p_{c 1 s}\right)$, which we know satisfied for all $\pi_{s}>\pi_{t}$. The first deviation cannot be profitable. The second deviation is ruled out if $v_{c}^{*}\left(p_{c B t}-p_{c 1 s}\right)>v_{c}\left(p_{c B t}-p_{c 1 s}\right)$ for all $v_{c} \leq v_{c}^{*}$. A sufficient condition then is $p_{c B t}>p_{c 1 s}$. it is not difficult to verify that $p_{c B t}>p$, while $p_{c 1 s}<p$, implying that the second deviation cannot be profitable either.

Finally, Proposition 5 states that the chair will order his $S$ lowest intensity proposals in the first $S$ positions of the agenda. Because the bonus vote is spent 
on $v_{c}^{*}$, the stated ordering is indeed a best response for the chair if $p_{c 1 t}>p_{c 1 s}$, which again is immediately verified, given $\pi_{t}<\pi_{s}<1 / 2$.

(2) Suppose now $n=3$. The logic proceeds identically. Again, consider the best response strategy for voter $i$, who is not the chair. Voter $i$ casts the bonus vote on proposal $P_{t}$ over proposal $P_{s}$ if the ratio $v_{i t} / v_{i s}$ is larger than:

$$
\frac{p_{B s}-p_{1 s}}{p_{B t}-p_{1 t}}=\frac{1-\pi_{s} \pi_{c s}}{1-\pi_{t} \pi_{c t}}
$$

where the right-hand side of (A.25) is derived taking into account $B \in \mathcal{B}(3)$. If $P_{s}$ and $P_{t} \in \mathbf{P}_{\mathcal{S}}$, or if $P_{s}$ and $P_{t} \in \mathbf{P}_{\mathcal{T}-\mathcal{S}}$, in the candidate equilibrium the ratio on the right-hand side of (A.25) equals 1: only $P_{i \mathcal{S}}^{*}$ and $P_{i \mathcal{T}-\mathcal{S}}^{*}$ are candidates for $i$ 's bonus vote. Suppose then $P_{t}=P_{i \mathcal{T}-\mathcal{S}}^{*}$ and $P_{s}=P_{i \mathcal{S}}^{*}($ and $S>0$ ). The ratio in (A.25) becomes:

$$
\frac{p_{B s}-p_{1 s}}{p_{B t}-p_{1 t}}=\frac{1}{1-\pi_{t} /(T-S)}
$$

Call the right-hand side of (A.25') $\delta\left(\pi_{t}\right)$. Voter $i$ 's best response is to cast the bonus vote on $P_{t}$ if and only if $v_{i \mathcal{T}-\mathcal{S}}^{*} \geq \delta\left(\pi_{t}\right) v_{i \mathcal{S}}^{*}$. Thus $\pi_{t}=\operatorname{prob}\left(v_{j t}=\right.$ $\left.v_{j \mathcal{T}-\mathcal{S}}^{*}\right) \operatorname{prob}\left(v_{j \mathcal{T}-\mathcal{S}}^{*}>\alpha v_{j \mathcal{S}}^{*}\right)$. In a quasi-symmetrical equilibrium, $\pi_{t}$ must solve:

$$
\pi_{t}=\int_{0}^{1}\left[F\left(\frac{v}{\delta\left(\pi_{t}\right)}\right)\right]^{S}[F(v)]^{T-S-1} d F(v) \equiv \zeta\left(\pi_{t}\right)
$$

Both $\delta$ and $\zeta$ are continuous in $\pi_{t}$. Note that $\lim _{\pi_{t \rightarrow>0}} \delta\left(\pi_{t}\right)=1$, and hence $\lim _{\pi_{t \rightarrow>0}} \zeta\left(\pi_{t}\right)=1 / T>0$; while $\left.\delta\left(\pi_{t}\right)\right|_{\pi_{1}=1 / T}>1$ for all finite $T$, and hence $\left.\zeta\left(\pi_{t}\right)\right|_{\pi_{t}=1 / T}<1 / T$. In addition, $\delta$ is everywhere increasing in $\pi_{t}$, and thus $\zeta$ is everywhere decreasing in $\pi_{t}$. It follows that $\zeta$ (A.26) must have a unique solution at some $\widetilde{\pi_{t}} \in(0,1 / T)$. Thus $\left.\delta\left(\pi_{t}\right)\right|_{\pi_{t}=\widetilde{\pi_{t}}} \equiv \alpha(3, G, T, S)>1$ for all $G, T, S$, with $S>0$. Note that for all $\delta\left(\widetilde{\pi_{t}}\right)>1, \zeta\left(\widetilde{\pi}_{t}\right)$ must be lower the higher is $S$, and thus for given $G$ and $T, \widetilde{\pi_{t}}$ must be lower the higher is $S$. As in the case of $n=4, \widetilde{\pi_{t}}$ is monotonically lower the more informative is the equilibrium.

Consider now the chair's problem. As argued earlier, given the order of the proposals and the other voters' posited strategies, the only possible targets for the chair's bonus vote are his highest intensity proposals in the two sets, $P_{c \mathcal{T}-\mathcal{S}}^{*}$ and $P_{c \mathcal{S}}^{*}$. Suppose then $P_{t}=P_{i \mathcal{T}-\mathcal{S}}^{*}$ and $P_{r}=P_{i \mathcal{S}}^{*}$, and recall that in the candidate equilibrium $v_{c \mathcal{T}-\mathcal{S}}^{*} \geq v_{c \mathcal{S}}^{*}$. With $B \in \mathcal{B}(3)$ and $n=3$ :

$$
\frac{p_{c B r}-p_{c 1 r}}{p_{c B t}-p_{c 1 t}}=\left(\frac{1-\pi_{r}^{2}}{1-\pi_{t}^{2}}\right)
$$

an expression smaller than 1 for all $\pi_{r}>\pi_{t}$. Thus with $v_{c \mathcal{T}-\mathcal{S}}^{*} \geq v_{c \mathcal{S}}^{*}$, it is indeed optimal for the chair to cast his bonus vote on $P_{i \mathcal{T}-\mathcal{S}}^{*}$. Can the chair do better by deviating in the order of the proposals? Once again, two deviations must be considered: (1) The chair could order his highest intensity proposal in the first $S$ positions on the agenda, and cast on it his bonus vote; (2) the chair 
could order his highest intensity proposal in the first $S$ positions, but cast his bonus vote on $P_{c \mathcal{T}-\mathcal{S}}^{*}$. Using the index $t$ to refer to a proposal in $\mathbf{P}_{\mathcal{T}-\mathcal{S}}$ and the index $s$ to refer to a proposal in $\mathbf{P}_{\mathcal{S}}$, the first deviation is ruled out if $v_{c}^{*}\left(p_{c B t}-\right.$ $\left.p_{c B s}\right)>v_{c}\left(p_{c 1 t}-p_{c 1 s}\right)$ for all $v_{c} \leq v_{c}^{*}$. A sufficient condition then is $\left(p_{c B t}-\right.$ $\left.p_{c 1 t}\right)>\left(p_{c B s}-p_{c 1 s}\right)$, a condition we know satisfied for all $\pi_{t}<\pi_{s}$. The first deviation cannot be profitable. The second deviation is ruled out if $v_{c}^{*}\left(p_{c B t}-\right.$ $\left.p_{c 1 s}\right)>v_{c}\left(p_{c B t}-p_{c 1 s}\right)$ for all $v_{c} \leq v_{c}^{*}$. A sufficient condition then is $p_{c B t}>$ $p_{c 1 s}$. But as in the case of $n=4, p_{c B t}>p$, while $p_{c 1 s}<p$, implying that the second deviation cannot be profitable either.

Finally, Proposition 5 states that the chair will order his $S$ lowest intensity proposals in the first $S$ positions of the agenda. Because the bonus vote is spent on $v_{c}^{*}$, the stated ordering is indeed a best response for the chair if $p_{c 1 t}>p_{c 1 s}$, which again is immediately verified with $\pi_{t}<\pi_{r}<1 / 2$ for all $T>2$.

(3) To show claim (2) in the proposition, it suffices to show that no other quasi-symmetrical equilibrium exists when $n=3$. In the equilibria characterized by the proposition: (i) If the chair randomizes over $P_{c}^{*}$ 's position in the agenda, he randomizes with equal probabilities. To show that this must be true, consider proposals in positions $r$ and $t$, and suppose $\pi_{c r}>\pi_{c t}>0$. With $n=3$, voter $i$ who is not chair prefers to cast his bonus vote on $P_{r}$ relative to some other proposal $P_{s}$ if $v_{i r} / v_{i s} \geq\left(1-\pi_{r} \pi_{c r}\right) /\left(1-\pi_{s} \pi_{c s}\right)$, and similarly for $P_{t}$. It then follows immediately that any quasi-symmetrical equilibrium where $\pi_{c r}>\pi_{c t}$ must have $\pi_{r}<\pi_{t}$. But for the chair this implies $\left(p_{c B r}-p_{c 1 r}\right)>\left(p_{c B t}-p_{c 1 t}\right)$ : in any realization where the chair orders $P_{c}^{*}$ as $P_{t}$, he can do strictly better by exchanging $P_{t}$ and $P_{r}$, and thus ordering $P_{c}^{*}$ as $P_{r}$ and casting his bonus vote on $P_{r}$. If $\pi_{c r}>\pi_{c t}$, then $\pi_{c t}=0 . \pi_{c r}>\pi_{c t}>0$ cannot be supported in equilibrium. (In fact, the same argument holds for $n=4$ if $T>2$ ). (ii) Given the chair's strategy, the only candidates for non-chair voter $i$ 's bonus vote are proposals $P_{i \mathcal{S}}^{*}$ and $P_{i \mathcal{T}-\mathcal{S}}^{*}$. To show that this must be true, again consider the ratio $\left(p_{c B r}-p_{c 1 r}\right) /\left(p_{c B t}-p_{c 1 t}\right)$. When both $P_{r}$ and $P_{t}$ belong to $\mathbf{P}_{\mathcal{S}}, \pi_{c r}=\pi_{c t}=0$; therefore with $n=3,\left(p_{c B r}-p_{c 1 r}\right) /\left(p_{c B t}-p_{c 1 t}\right)=1$, and $i$ 's best response is to cast the bonus vote on $P_{r}$ rather than $P_{t}$ if and only if $v_{i r}>v_{i t}$. The conclusion follows. When both $P_{r}$ and $P_{t}$ belong to $\mathbf{P}_{\mathcal{T}-\mathcal{S}}$, $\pi_{c r}=\pi_{c t}=1 /(T-S)$. Suppose $\pi_{r}>\pi_{t}$; then $\left(p_{c B r}-p_{c 1 r}\right) /\left(p_{c B t}-p_{c 1 t}\right)<$ 1 , and voter $i$ 's best response results in casting the bonus vote on $P_{t}$ more frequently than on $P_{r}$ : any quasi-symmetrical equilibrium requires $\pi_{r}=\pi_{t}$, and thus $\left(p_{c B r}-p_{c 1 r}\right) /\left(p_{c B t}-p_{c 1 t}\right)=1$, and the conclusion follows. Thus for $n=3$, the only payoff-distinct informative equilibria are the ones described in the proposition, and claim (2) is established.

Proof of Proposition 6. In all informative equilibria, $S>0$, and:

$$
E V_{c}^{S}=\left(p_{c B t}-p_{c 1 t}\right) E v_{(T)}+\sum_{r=1}^{S} E v_{(r)}\left(p_{c 1 s}-p_{c 1 t}\right)+T E v p_{c 1 t}
$$

with the probabilities amended to take into account the voting strategies of the other committee members and derived above in the proofs to Propositions 4 
and 5. Propositions 4 and 5 imply $\pi_{t} \leq 1 / T$ and $\pi_{s}=\left[1-(T-S) \pi_{t}\right] / S \geq 1 / T$. If $\pi_{t}=1 / T$, then $\pi_{s}=1 / T, p_{c 1 s}=p_{c 1 t}$ and $E V_{c}^{S}=E V_{c}^{N}$. Thus the proof of the proposition amounts to showing that $E V_{c}^{S}$ is strictly declining in $\pi_{t}$ for all $\pi_{t} \in\left[\underline{\pi_{t}}, 1 / T\right]$ where $\pi_{t}$ is the lower bound on possible equilibrium values of $\pi_{t}$. Because $\pi_{t}$ and the probabilities $p_{c B t}, p_{c 1 t}$, and $p_{c 1 s}$ depend on $n, T, S$, and $B$ the different cases need to be studied separately. The proof is tedious but straightforward.

(i) Consider first the equilibria described in Proposition 4, where $T=2$ and $S=1$. (A.27) can be rewritten as:

$$
E V_{c}^{S}=\left(p_{c B 1}-p_{c 1 s}\right) E v_{(T)}+T p_{c 1 s} E v .
$$

As shown in the proof to Proposition 4 , if $n$ is even $\alpha(n, G, S)=1$, or correspondingly $\pi_{1}=1 / T$. Thus $E V_{c}^{F I}=E V_{c}^{N}=E V_{c}$ for all $n$ even. Consider then $n$ odd. Substitute $p_{c B 1}$ and $p_{c 1 s}$ in (A.28) from (A.20) and (A.21), taking into account that $T=2$, and thus $\pi_{2}=1-\pi_{1}$. Differentiating with respect to $\pi_{1}$, we obtain:

$$
\frac{\partial E V_{c}^{S}}{\partial \pi_{1}}<0 \Longleftrightarrow \frac{E v_{(2)}}{E v}>\frac{2(n+1) \pi_{1}\left(2 \pi_{1}-1\right)}{(n+1) \pi_{1}\left(2 \pi_{1}-1\right)+\left(1-\pi_{1}\right)[3-n+2(n-1)]} .
$$

The condition is always satisfied if the ratio on the right-hand side of (A.29) is smaller than 1. But:

$$
\frac{2(n+1) \pi_{1}\left(2 \pi_{1}-1\right)}{(n+1) \pi_{1}\left(2 \pi_{1}-1\right)+\left(1-\pi_{1}\right)[3-n+2(n-1)]}<1 \Longleftrightarrow 4 \pi_{1}-3+n\left(1-2 \pi_{1}\right)^{2}<0 .
$$

This latter inequality is guaranteed satisfied if $\pi_{1} \in[((n-1)-\sqrt{n+1}) /(2 n), 1 / 2]$. The proof of Proposition 4 establishes a lower bound $\pi_{1}$ on $\pi_{1}$, where $\pi_{1}$ is the solution to $\beta\left(\underline{\pi_{1}}\right)=1$ (and thus $\varphi\left(\underline{\pi_{1}}\right)=1 / 2$ ). Solving $\beta\left(\underline{\pi_{1}}\right)=1$, we obtain:

$$
\underline{\pi_{1}}=\frac{1}{4}[3-n+\sqrt{(n-3)(n+1)}]>\frac{(n-1)+\sqrt{n+1}}{2 n} .
$$

Thus the ratio on right-hand side of (A.29) is smaller than 1 for all $\pi_{1} \in\left[\pi_{1}, 1 / 2\right]$, implying that $E V_{c}^{I}$ is declining in $\pi_{1}$ over all values of $\pi_{1}$ in that range, and thus $E V_{c}^{S}\left(\widetilde{\pi}_{1}\right)>\left.E V_{c}^{S}\right|_{\pi_{1}=1 / 2}=E V_{c}$.

(ii) Consider now the equilibria described in Proposition 5, where $n \in\{3,4\}$ and $B \in \mathcal{B}(n)$. Suppose first $n=3$. With $n=3$ and $B \in \mathcal{B}(n)$ :

$$
\begin{aligned}
p_{c B t}-p_{c 1 t} & =\frac{\left(1-\pi_{t}\right)^{2}}{8}+\frac{\pi_{t}\left(1-\pi_{t}\right)}{4} \\
p_{c 1 s}-p_{c 1 t} & =\frac{\pi_{t}\left(1-\pi_{t}\right)}{4}-\frac{\pi_{s}\left(1-\pi_{s}\right)}{4} \\
p_{c 1 t} & =(3 / 4)-\frac{\pi_{t}\left(1-\pi_{t}\right)}{4}
\end{aligned}
$$


and recall that $\pi_{s}=\left[1-(T-S) \pi_{t}\right] / S$. Differentiating (A.27) with respect to $\pi_{t}$, we obtain:

$\frac{\partial E V_{c}^{S}}{\partial \pi_{t}}=\frac{1}{4 S^{2}}\left[-E v_{(T)} \pi_{t} S^{2}+E v\left(2 \pi_{t}-1\right) S^{2} T+\sum_{r=1}^{S} E v_{(r)}\left[S\left(2+T-4 \pi_{t} T\right)+2 T\left(\pi_{t} T-1\right)\right]\right]$

It is not difficult to verify that $\partial^{2} E V_{c}^{S} / \partial \pi_{t}^{2}>0$, and thus (A.30) is maximal at $\pi_{t}=1 / T$. But:

$$
\left.\frac{\partial E V_{c}^{S}}{\partial \pi_{t}}\right|_{\pi_{t}=1 / T}=\frac{-1}{4 S^{2}}\left[S E v_{(T)}+T(T-2)\left(S E v+\sum_{r=1}^{S} E v_{(r)}\right)\right]<0
$$

and thus (A.30) is negative for all $\pi_{t} \in[0,1 / T]$. Notice that although we know that $\pi_{t}$ is smaller the higher is $S$, the observation does not imply that (A.27) must be higher the higher is $S$ because at higher $S$ the number of terms in the summation increase, and $\left(p_{c 1 s}-p_{c 1 t}\right)<0$ for all $\pi_{t}<1 / T$.

Suppose now $n=4$. With $n=4$ and $B \in \mathcal{B}(n)$ :

$$
\begin{gathered}
p_{c B t}-p_{c 1 t}=\frac{3}{16}\left[1-\pi_{t}\left(1-\pi_{t}\right)\right] \\
p_{c 1 s}-p_{c 1 t}=\frac{3}{16} \pi_{t}\left[1-\pi_{t}\left(1-\pi_{t}\right)\right]-\frac{3}{16} \pi_{s}\left[1-\pi_{s}\left(1-\pi_{s}\right)\right] \\
p_{c 1 t}=(11 / 16)-\frac{3}{16} \pi_{t}\left[1-\pi_{t}\left(1-\pi_{t}\right)\right]
\end{gathered}
$$

Differentiating (A.27) with respect to $\pi_{t}$, we obtain:

$$
\frac{\partial E V_{c}^{S}}{\partial \pi_{t}}=\frac{1}{16}\left[-3 E v_{(T)}\left(1-2 \pi_{t}\right)-3 T E v\left(1-2 \pi_{t}+3 \pi_{t}^{2}\right)+\frac{1}{S^{3}} \sum_{r=1}^{S} E v_{(r)} A\left(\pi_{t}\right)\right]
$$

where:

$$
\begin{aligned}
A\left(\pi_{t}\right) \equiv & 9 T\left(1-\pi_{t} T\right)^{2}+3 S\left(1-\pi_{t} T\right)\left(9 \pi_{t} T-3-2 T\right)+ \\
& +3 S^{2}\left[2+T-\pi_{t}\left(6+4 T-9 \pi_{t} T\right)\right]
\end{aligned}
$$

If $A\left(\pi_{t}\right)<0$, then $\partial E V_{c}^{S} / \partial \pi_{t}<0$ and the proof is concluded. If $A\left(\pi_{t}\right)>0$, then $\partial E V_{c}^{S} / \partial \pi_{t}$ is increasing in $\sum_{r=1}^{S} E v_{(r)}$. But $\sum_{r=1}^{S} E v_{(r)}<S E v$, and thus $\partial E V_{c}^{S} / \partial \pi_{t}$ must be negative if:

$$
-3 E v_{(T)}\left(1-2 \pi_{t}\right)-E v\left[3 T\left(1-2 \pi_{t}+3 \pi_{t}^{2}\right)-\frac{1}{S^{2}} A\left(\pi_{t}\right)\right]<0
$$

Manipulating this expression, we obtain $3 T\left(1-2 \pi_{t}+3 \pi_{t}^{2}\right)-\frac{1}{S^{2}} A\left(\pi_{t}\right)>0 \Longleftrightarrow$ $3-2 S-3 \pi_{t}(T-2 S)<0$. It is not difficult to see that the condition must be satisfied for all $S>1$. When $S=1$, the condition requires $\pi_{t}>1 /(3 T-6)$. But if $S=1, \underline{\pi_{t}}=1 /(1+\sqrt{1+T(T-1)})>1 /(3 T-6)$ for all $T>3$. The only 
remaining case is then $S=1, T=3$. But it is then immediate to verify that for $S=1, T=3, \partial E V_{c}^{S} / \partial \pi_{t}<0$ for all $\pi_{t}>0.131$, a condition that must be satisfied for all $\pi_{t} \in\left[\underline{\pi_{t}}, 1 / T\right]$ since $\underline{\pi_{t}}=1 /(1+\sqrt{7})=0.274$. Thus (A.31) must be always negative for all $\pi_{t} \in\left[\pi_{t}, \overline{1} / T\right]$, and the proof is concluded.

Equilibria of the experimental treatments. (1). The existence of the NI and PI equilibria, and of the FI equilibrium where the chair orders $P_{c}^{*}$ as the third proposal follows from Proposition 5. The FI equilibrium where $P_{c}^{*}$ is ordered second mirrors the payoff-equivalent equilibrium where $P_{c}^{*}$ is ordered third - voting choices over the last proposal are residual, and the chair cannot affect any non-chair strategy by deviating off equilibrium and not casting the bonus vote on $P_{2}$. The FI equilibrium where $P_{c}^{*}$ is ordered first does require the specification of off-equilibrium beliefs that can support it as a sequential equilibrium. Plausible beliefs $\pi_{c 2}=\pi_{c 3}=1 / 2$, were the chair to deviate and not cast the bonus vote on $P_{1}$, are sufficient for the purpose: they guarantee $\pi_{2}>\pi_{1}$ and thus support the chair's equilibrium strategy. (2) Showing that with the experimental parameterization no other equilibrium exists is more cumbersome, but again builds on arguments used in the proofs to the propositions. For $n=3$, the proof of Proposition 1 shows that no other NI equilibrium exists; the proof of Proposition 5 shows that in the PI equilibrium the chair must randomize the order of $P_{c}^{*}$ over positions $\{T-S, . ., T\}$ with equal probability, and that in all equilibria the only candidates for non-chair voter $i$ 's bonus vote are proposals $P_{i \mathcal{S}}^{*}$ and $P_{i \mathcal{T}-\mathcal{S}}^{*}$. When $T=3$, all results hold true for $n=4$ and because the proofs follow the identical logic, I do not repeat them here. These results establish that no other payoff-distinct quasi symmetrical informative equilibrium exists; that the NI equilibrium is unique, and that all FI equilibria are as described. To establish that the list of equilibria in the text is exhaustive, then, one result is missing: we need to show that with $T=3$ and $n \in\{3,4\}$, the PI equilibrium remains unique when we allow for payoff-equivalent equilibria. This is the purpose of the following Lemma:

Lemma: With $T=3$ and $n \in\{3,4\}$, there is a unique PI equilibrium, and in such equilibrium the chair orders his lowest intensity proposal as $P_{1}$ : $\mathbf{P}_{\mathcal{T}-\mathcal{S}}=\left\{P_{2}, P_{3}\right\}$.

Proof. Suppose to the contrary that $\mathbf{P}_{\mathcal{T}-\mathcal{S}}=\left\{P_{1}, P_{3}\right\}$ : the chair orders his lowest intensity proposal as $P_{2}$, and casts his bonus vote with equal probability on $P_{1}$ and $P_{3}: \pi_{c 1}=\pi_{c 3}=1 / 2 ; \pi_{c 2}=0$. If such an equilibrium exists, a nonchair voter must cast his bonus vote either on $P_{2}$ or on $P_{\mathcal{T}-\mathcal{S}}^{*}$ which is equally likely to be $P_{1}$ or $P_{3}$. Hence, with $n=3$, and following (A25'): $\pi_{1}=\operatorname{prob}\left(v_{i 1}=\right.$ $\left.\max \left\{v_{i 1}, v_{i 3}\right\}\right) \operatorname{prob}\left(\max \left\{v_{i 1}, v_{i 3}\right\}>\left(2 v_{i 2}\right) /\left(2-\pi_{1}\right)\right)$. But if the chair's bonus vote is not cast on $P_{1}$, then, under the candidate strategy, it must be cast on $P_{3}$ with probability 1 . Non-chair voters will then cast their bonus vote on $P_{3}$ with probability $\pi_{3}^{n}=\operatorname{prob}\left(v_{i 3}=\max \left\{v_{i 1}, v_{i 3}\right\}\right) \operatorname{prob}\left(\max \left\{v_{i 1}, v_{i 3}\right\}>v_{i 2} /\left(1-\pi_{3}^{n}\right)\right)$, where the superscript $n$ indicates that the probability has been updated after the chair failed to cast his bonus vote on $P_{1}$. But then $\pi_{3}^{n}<\pi_{1}$ (in the Uniform parameterization $\pi_{3}^{n}=1 / 4$ and $\pi_{1}=2 / 7$ ): the chair can gain from deviating and always ordering $P_{c}^{*}$ as $P_{3}$, and the scenario cannot be an equilibrium. With 
$n=4$, the relevant probabilities are given by (A.23'), but the logic is identical (in the Uniform parameterization $\pi_{3}^{n}=0.274$ and $\pi_{1}=0.307$ ). Finally, the same logic also establishes that there cannot be an equilibrium with $\mathbf{P}_{\mathcal{T}-\mathcal{S}}=\left\{P_{1}, P_{2}\right\}$. 


\section{$9 \quad$ Appendix B. Sample instructions $(n=3)$}

\subsection{General Instructions}

This is an experiment in group decision making. By following the instructions and making careful decisions you will earn varying amounts of money, which will be paid at the end of the experiment. Your earnings will depend partly on your decisions, partly on the decisions of others, and partly on chance.

The entire experiment will take place through computer terminals, and your interaction with other participants will take place through the computers. It is important that you do not talk or in any way try to communicate with other participants during the experiment.

We will start with a brief instruction period. During the instruction period, you will be given a complete description of the experiment and will be shown how to use the computers. If you have any questions during the instruction period, raise your hand and your question will be answered out loud so that everyone can hear. If any difficulties arise after the experiment has begun, raise your hand, and an instructor will come and assist you.

\subsection{Decision Problem}

The decision problem you are participating in is about voting. In this experiment, you will participate in 40 independent voting sessions. At the beginning of the experiment you will be randomly assigned to a group of three people. This will be your group for the entire experiment. Each group follows exactly the same rules and what happens in one group has no effect on the other groups.

In each session, you and the other members of your group will be asked to vote over three successive proposals, each of which can either pass or fail. The proposals are called Green Proposal, Blue Proposal, and Red Proposal. Each member of a group has one regular vote to be cast for each proposal. In addition, each member will be given one extra bonus vote. You will choose in which of the three proposals you want to cast the bonus vote in addition to your regular vote.

At the beginning of a session you will be assigned a personal Green Proposal Value, Blue Proposal Value, and Red Proposal Value. Each of the three values is equally likely to be any integer amount between -100 and 100 , but not zero. The three values are assigned randomly and thus will generally be different. The other members of your group are also randomly assigned their own Green Proposal Values, Blue Proposal Values, and Red Proposal Values each of which is equally likely to be any integer amount between -100 and 100 , but not zero. Because the values are random, each person is likely to have different values for the three proposals, and the values of each proposal (for example the Green Proposal Values) are also likely to be different for three different persons. You are not told what the values of the other members of your group are.

If your value is positive, you are in favor of the proposal and earn your value if and only if the proposal passes. If your value is negative, you are against the 
proposal and earn the absolute value of your value if and only if the proposal does not pass. Otherwise you earn 0 francs. For example, if your Green Proposal Value is -55 , then you earn 55 francs if Green does not pass, and 0 francs if Green passes (and similarly for Blue and Red proposals). Because the values are randomly assigned, being in favor of one proposal carries no implication for how you feel about the other.

As mentioned earlier, the three elections are held sequentially. Each member of the group will cast one regular vote in each proposal. In addition, each member will be given one extra bonus vote and will choose in which of the three proposals s/he wants to cast the bonus vote in addition to his/her regular vote. Suppose for example that the first election is on the Blue proposal. Then you will decide whether to cast 1 or 2 votes in the Blue election: your regular Blue vote only, or your regular Blue vote and the bonus vote. The other members of your group may also cast up to 2 votes in the Blue election. If you are in favor of the Blue proposal (that is, your Blue Proposal Value is positive), any votes you cast will automatically be counted as YES votes. If you are against Blue (that is, your Blue Proposal Value is negative), any votes you cast will be automatically counted as NO votes. The Blue proposal passes in your group if there are more YES votes than NO votes cast by members of your group and fails if there are more NO votes than YES votes. Ties are broken randomly by the computer.

After you and the other members of your group have made voting decisions for the first proposal, the computer will tell you the number of votes cast for and against the proposal, and the outcome of the vote for your group. You will then proceed to the second proposal.

Continuing with the example, suppose that the second election is the Green election. In the Green election each member of your group (including you) have his or her regular green vote, plus the bonus vote, unless the bonus vote was cast earlier in the Blue election. If you cast 2 votes in the Blue election, you will have only 1 vote in the Green election; but if you cast 1 vote in the Blue election, you will have up to 2 votes in the Green election. Your screen will display how many votes you and the other members of your group have left.

Again, after you and the other members of your group have made voting decisions for the Green proposal, the computer will tell you the number of votes cast for and against the proposal, and the outcome of the vote for your group. You will then proceed to the third proposal.

Your screen will display how many votes you and the other members of your group have left for the third proposal, the Red proposal in the example. You do not have any choice about how many votes to cast in this third election, but by clicking the VOTE button, you will cast all your remaining votes. These votes will automatically be cast as YES vote for the Red Proposal if your Red Proposal Value is greater than 0 and as NO vote if your Red Proposal Value is less than 0. Your group's outcome of the Red election is then reported to you.

When everyone has finished we will go to the next voting session, that is to another set of three sequential elections. You will repeat the procedure described above for 10 sessions. 
In the first 10 sessions, after all personal values have been assigned, the computer randomly chooses the order in which the three proposals will be voted, and you will be informed about this order. In the following 10 sessions (i.e. sessions 11-20), after all personal values have been assigned, the computer randomly assigns a member of the group to be the Chair for the next 10 sessions. The Chair decides the order in which the three elections will be held. You will see on your computer monitor whether you have been chosen to be the Chair. If so, you will be asked to choose the order of the proposals; if not you will be told what order of elections the Chair has chosen. Voting then proceeds as we described earlier. After the 20th session, the computer will again randomly assign one of the two other members of the group as chair for the next 10 sessions (i.e. sessions 21-30) and the same procedure will take place. Finally, the last member of the group will be the chair in the last 10 sessions (i.e. sessions 31-40.)

\subsection{Payoffs}

Everyone will be paid in private and you are under no obligation to tell others how much you earned. Your earnings during the experiment are denominated in francs. At the end of 40 voting sessions, the computer will randomly select one from sessions 1-10, 11-20, 21-30 and 31-40. The sum of your earnings in these four sessions will determine your total earning. Your dollar earnings are determined by dividing your earnings in francs by a conversion rate. In this experiment, the conversion rate is 18 , meaning that

$$
\$ 1=18 \text { francs, }
$$

This amount will then be added to the $\$ 7.00$ participation fee to determine your final payment. Payments will be made in private via petty cash vouchers at the conclusion of the experiment.

\subsection{Rules}

Please do not talk with anyone during the experiment. We ask everyone to remain silent until the end of the last decision problem.

Your participation in the experiment and any information about your earnings will be kept strictly confidential. Your receipt of payment and consent form are the only places on which your name will appear. This information will be kept confidential in the manner described in the consent form.

If you have any questions please ask them now. If not, we will have a practice round before we proceed to the paid session. 
Figure 1

Share of full efficiency as function of the number of voters

G Uniform, $T=3, B=1$

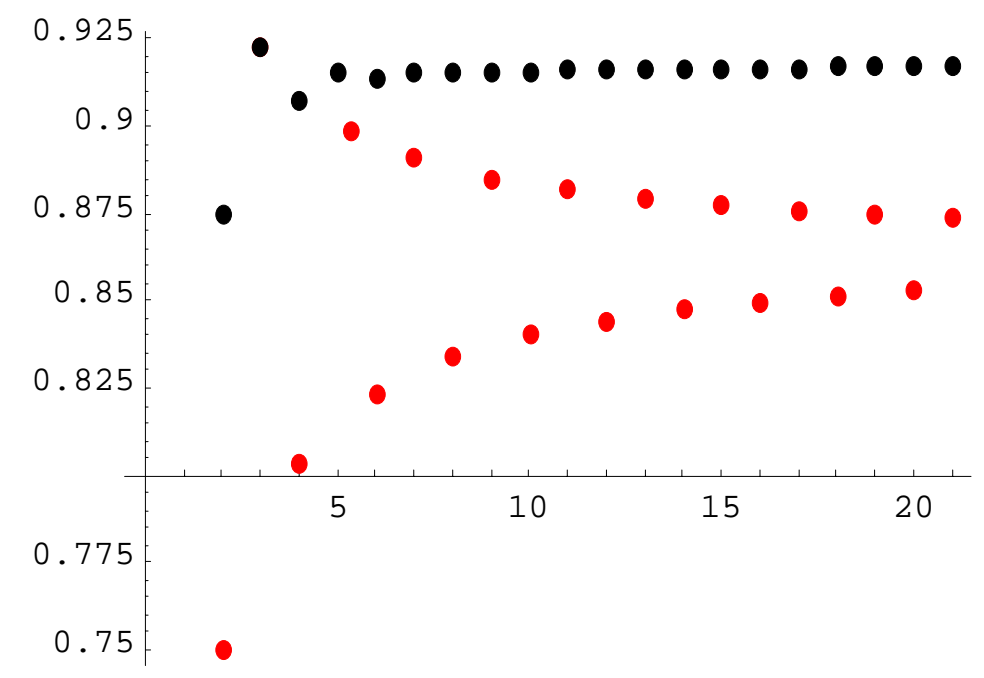

The black dots correspond to storable votes, and the red dots to simple majority. 
Figure 2. Expected utility in the fully informative equilibrium $\mathrm{G}$ Uniform, $\mathrm{n}=3$ and $\mathrm{n}=4, \mathrm{~B}=1$.

Figure 2a: Relative to the non-informative equilibrium, as function of $\mathbf{T}$.

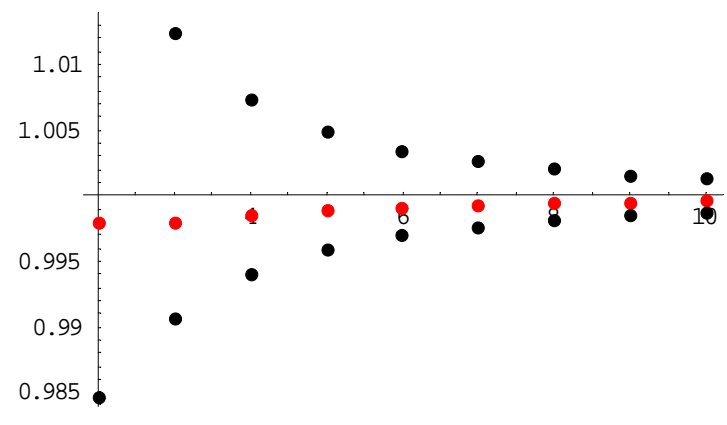

$\mathbf{n}=\mathbf{3}$

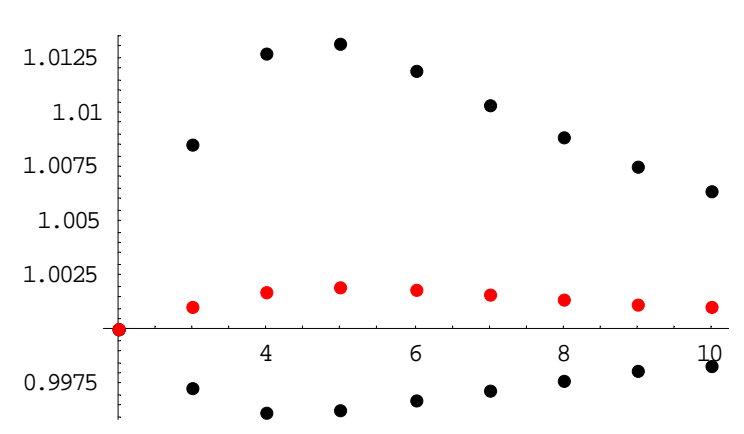

$n=4$

Figure 2b.Relative to expected utility with majority voting, as function of $T$.

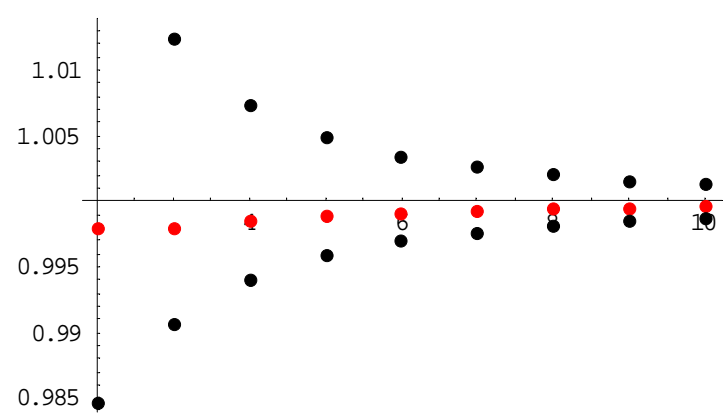

$\mathbf{n}=3$

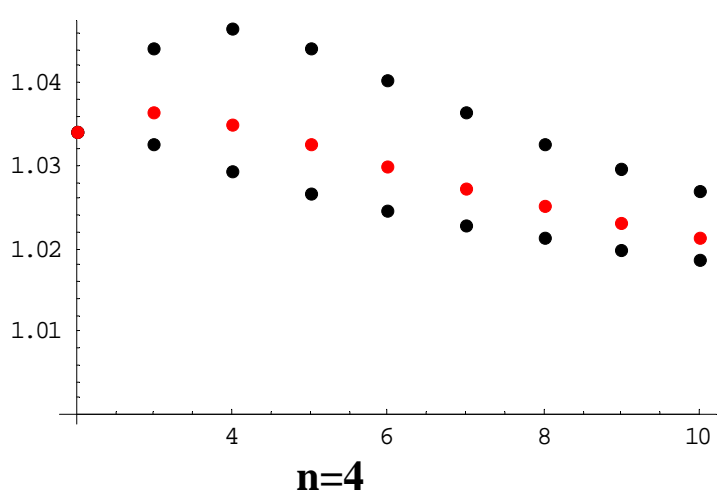

The upper black dots refer to the chair; the lower black dots to a representative voter who is not the chair, and the red dots to ex ante expected welfare with equal probability of being chair. 
Figure 3

The chair's ordering strategy.

Fraction of chairs whose ordering is always consistent with informative equilibria.

Figure 3a: All data

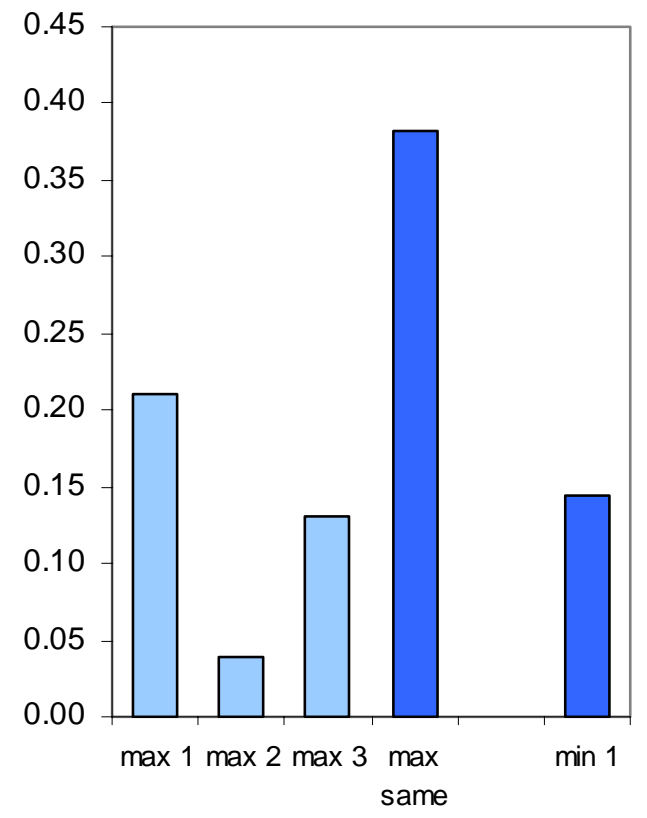

Figure 3b: With learning

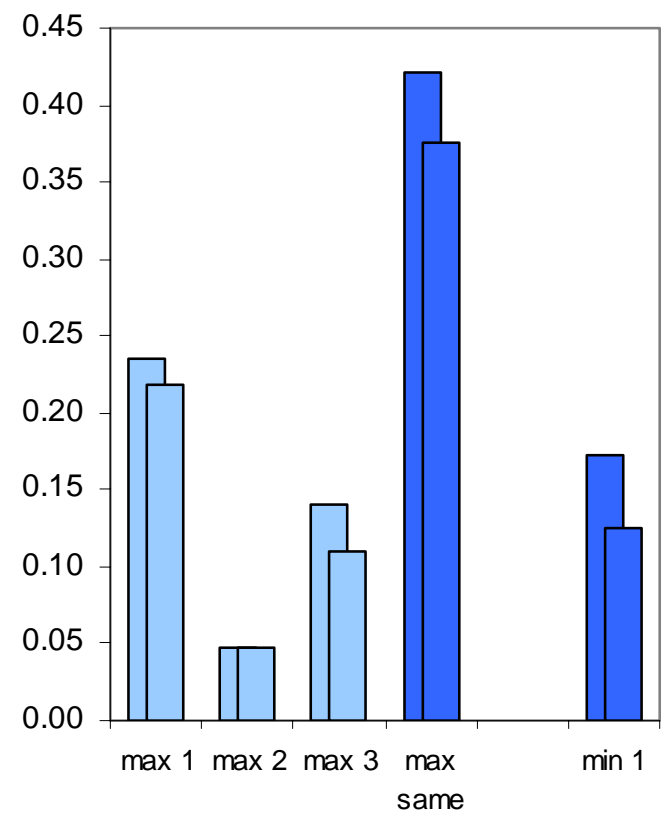


Figure 4

Aggregate error rates, by treatment and equilibrium.

Figure 4a

Full sample

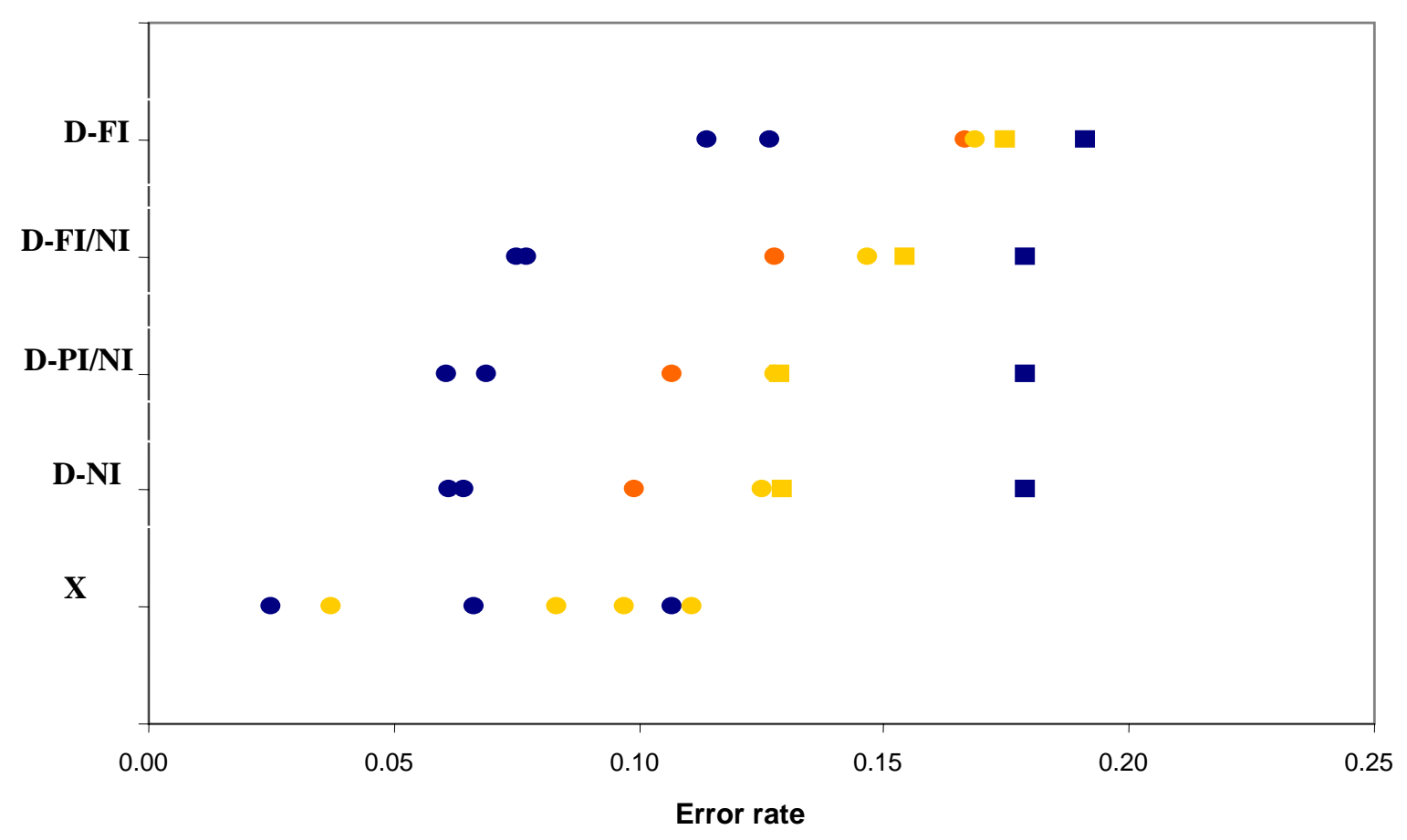

Figure 4b

Subsamples

Learning 1

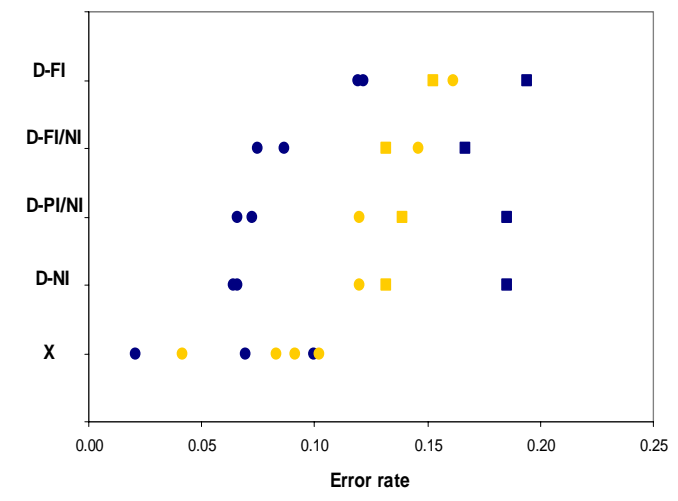

Learning 2

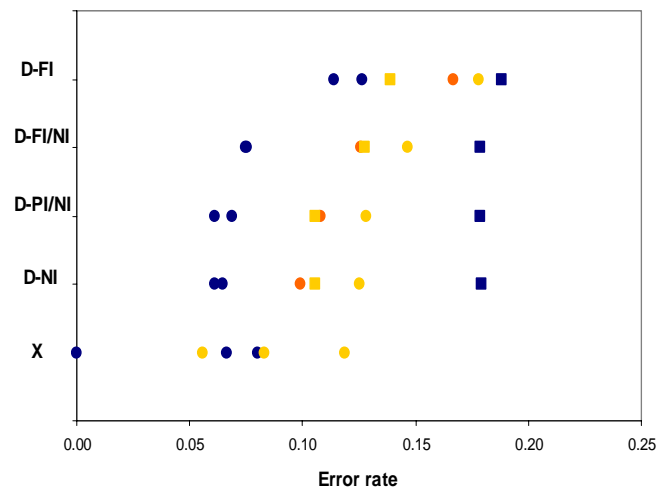

Learning 3

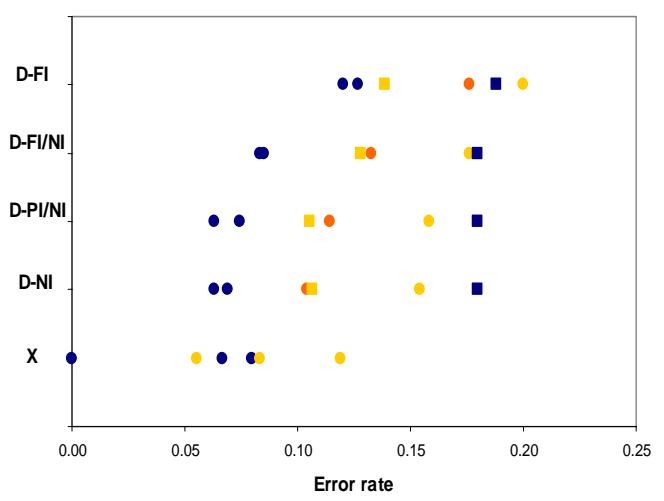


Figure 5

Histograms of individual error rates

Exogenous order (91 subjects)

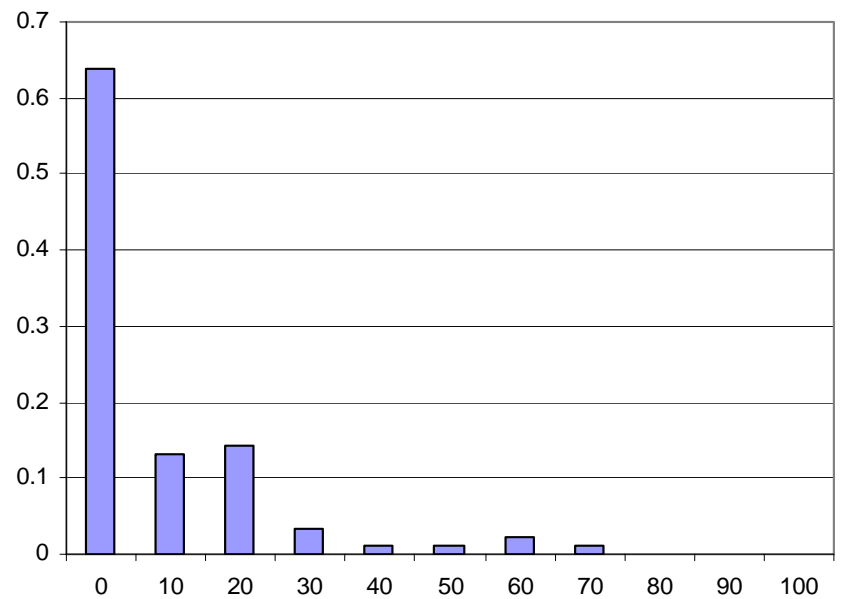

Endogenous order (74 subjects)

Non-informative equilibrium

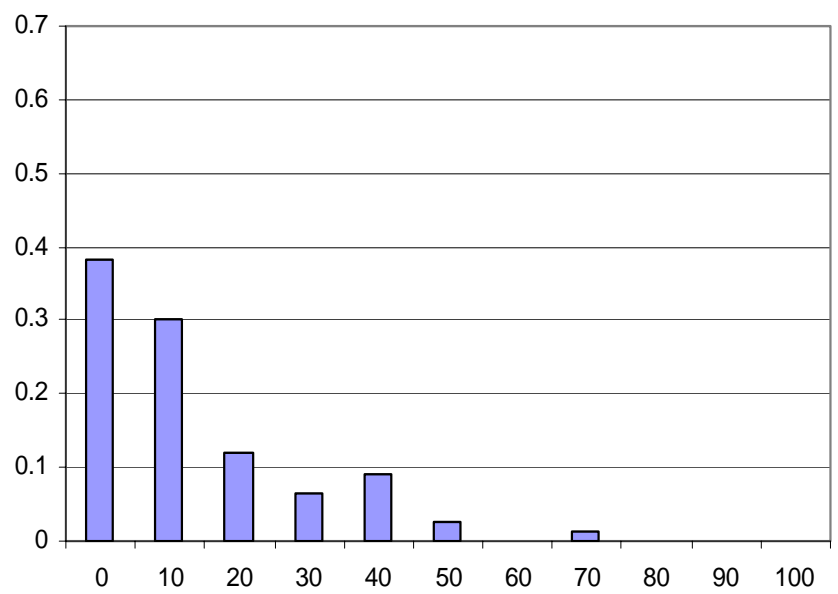

NI/FI equilibrium

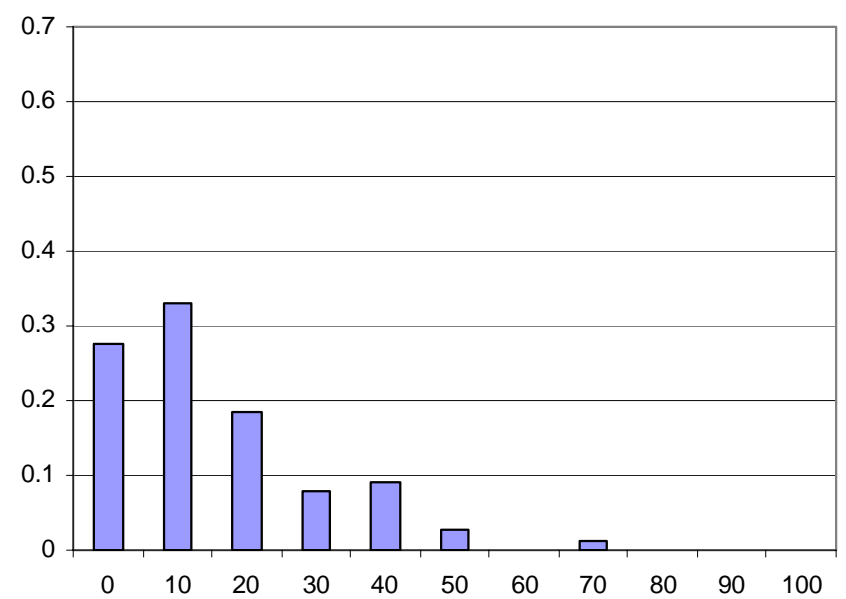

NI/PI equilibrium

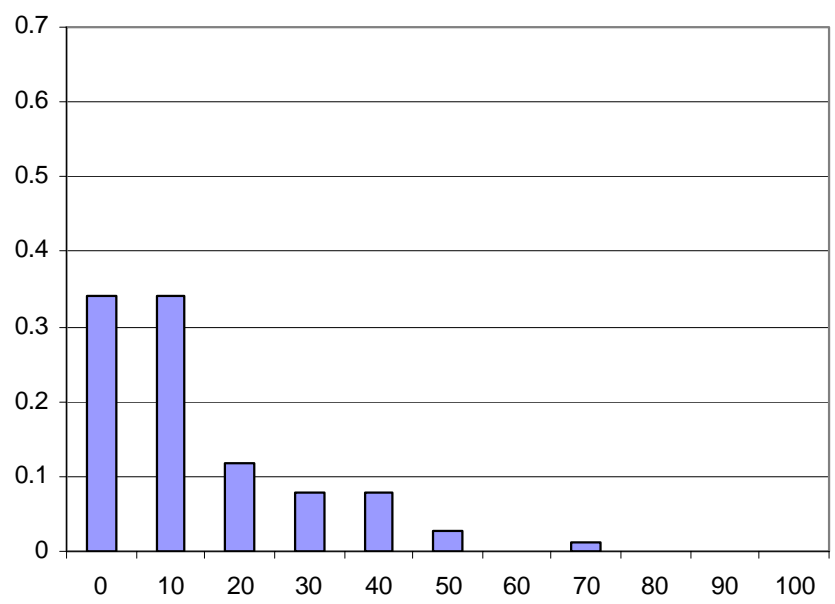

Fully informative equilibrium

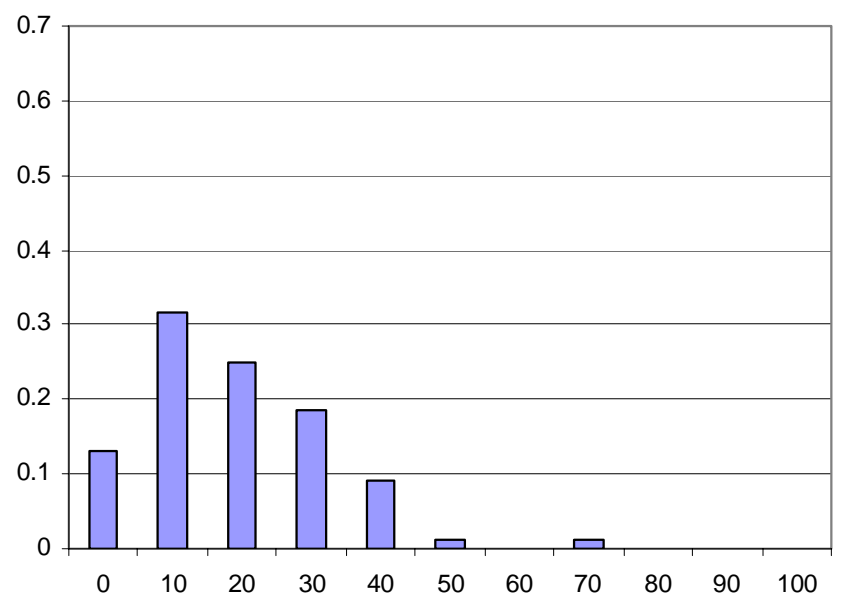


Figure 6

Experimental payoff (as share of available surplus)

Figure 6a: Experiment vs. equilibrium

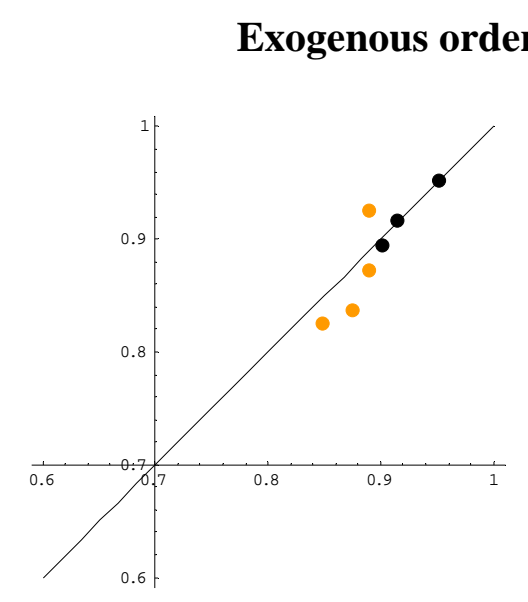

Endogenous order: FI equilibrium

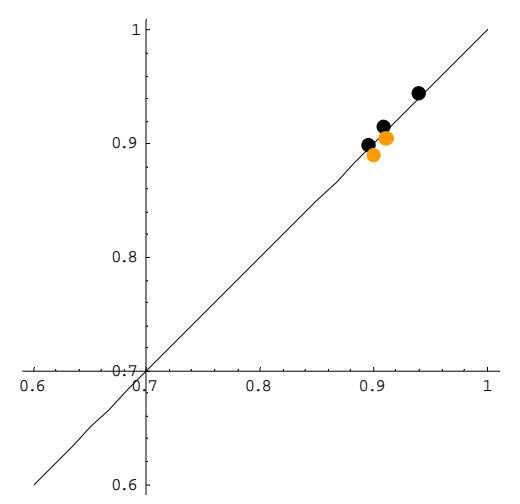

Endogenous order: NI equilibrium

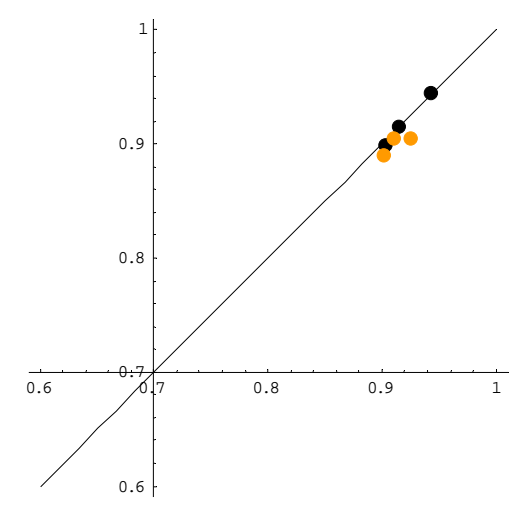

Endogenous order: PI equilibrium

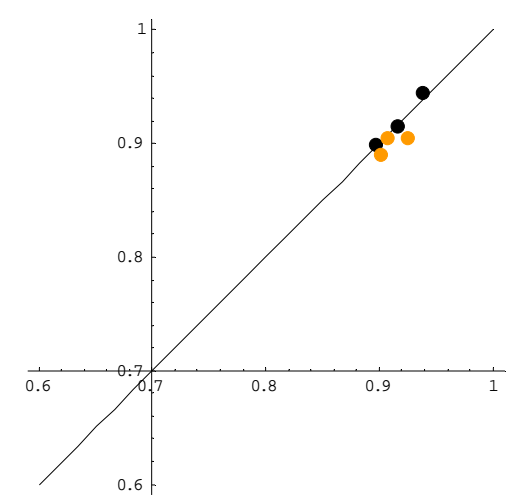

Figure 6b: Experiment vs. majority voting

Exogenous order

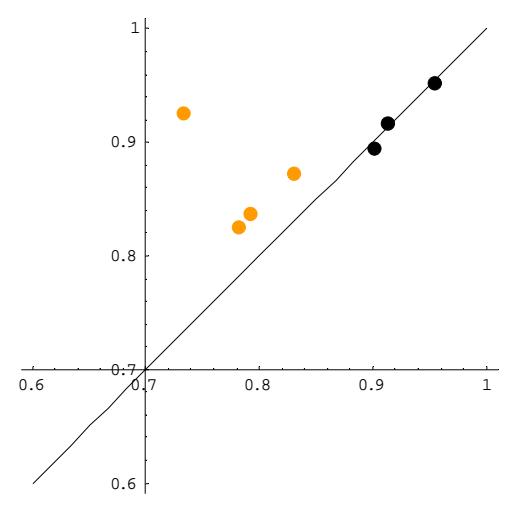

Endogenous order

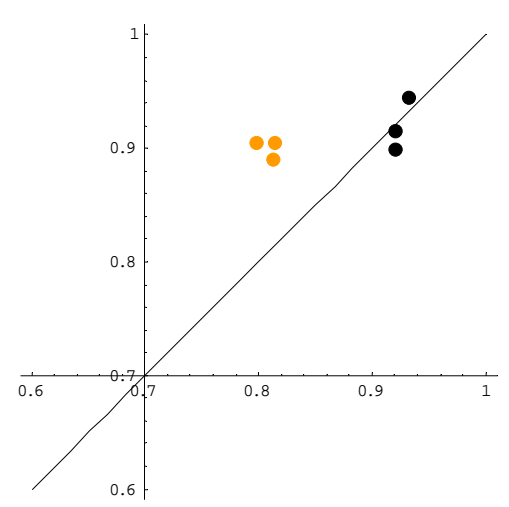

